\title{
A novel weighting switch function for uniformly high-order hybrid shock-capturing schemes
}

\author{
Jun Peng and Yiqing Shen*,† \\ State Key Laboratory of High Temperature Gas Dynamics, Institute of Mechanics, Chinese Academy of Sciences, \\ Beijing, China, 100190
}

\begin{abstract}
SUMMARY
Hybrid schemes are very efficient for complex compressible flow simulation. However, for most existing hybrid schemes in literature, empirical problem-dependent parameters are always needed to detect shock waves and hence greatly decrease the robustness and accuracy of the hybrid scheme. In this paper, based on the nonlinear weights of the weighted essentially non-oscillatory (WENO) scheme, a novel weighting switch function is proposed. This function approaches 1 with high-order accuracy in smooth regions and 0 near discontinuities. Then, with the new weighting switch function, a seventh-order hybrid compact-reconstruction WENO scheme (HCCS) is developed. The new hybrid scheme uses the same stencil as the fifth-order WENO scheme, and it has seventh-order accuracy in smooth regions even at critical points. Numerical tests are presented to demonstrate the accuracy and robustness of both the switch function and HCCS. Comparisons also reveal that HCCS has lower dissipation and less computational cost than the seventh-order WENO scheme. Copyright (C) 2016 John Wiley \& Sons, Ltd.
\end{abstract}

Received 25 August 2015; Revised 31 May 2016; Accepted 18 July 2016

KEY WORDS: WENO scheme; switch function; hybrid scheme; compressible flow

\section{INTRODUCTION}

Compressible turbulent flow is characterized by hierarchies of length and time scales, which cover a very broad bandwidth, large gradients of flow variables in boundary layers and shear layers, and discontinuities at high Mach numbers. Therefore, a desired numerical scheme for direct and large eddy simulation of compressible turbulent flow should have high spectral resolution as well as the shock-capturing capability.

The weighted essentially non-oscillatory (WENO) finite difference scheme [1,2] has been widely used in complex flow simulation because of its high-order accuracy and its capability of resolving discontinuities without numerical oscillations. Within the general framework for smoothness indicator and nonlinear weight design given by Jiang and Shu [2], many efforts were made to improve the accuracy of the WENO scheme. Henrick et al. [3] suggested a mapping function to improve the accuracy of the WENO scheme at critical points. Borges et al. $[4,5]$ introduced the WENO$\mathrm{Z}$ scheme, which calculates the nonlinear weights with a higher order smoothness indicator. The WENO-Z scheme has lower dissipation and higher resolution than the classical WENO scheme of Jiang and Shu [2], and its computational cost is less than the mapping function method. The accuracy of the WENO-Z scheme was further improved by Yamaleev and Carpenter [6, 7] and Fan et al. [8] by introducing higher order smoothness indicators. Shen and Zha [9] showed that at transitional points, which connect a smooth region and a discontinuity, the accuracy of fifth-order WENO

\footnotetext{
*Correspondence to: Yiqing Shen, State Key Laboratory of High Temperature Gas Dynamics, Institute of Mechanics, Chinese Academy of Science, Beijing, China, 100190.

${ }^{\dagger}$ E-mail: yqshen@imech.ac.cn 
schemes is second order, and a multi-step weighting method $[10,11]$ was developed to improve the accuracy. Except for these improvements for the fifth-order WENO scheme, higher order WENO schemes (higher than fifth order) were also developed [12-14]. However, even though the order of accuracy for WENO schemes can be designed to be arbitrarily high, the spectral resolution of WENO schemes is still not satisfactory [15-17]. Particularly, their excessive numerical dissipation for small-scale structures in compressible turbulent flows may overwhelm physical dissipation [18].

Compact schemes [19] are attractive because of their spectral-like resolution. However, they generate numerical oscillations near shocks and large gradients and thus can not be directly applied to compressible flow simulation. In order to develop shock-capturing compact schemes, Deng et al. [20] developed the compact high-order nonlinear schemes by using the WENO scheme to calculate cell interface fluxes for a central compact scheme. Jiang et al. [21] developed the weighted compact scheme by substituting the low-order subschemes of the WENO scheme with low-order compact schemes. Ghosh and Baeder [22] proposed the conservative fifth-order compact-reconstructed WENO (CRWENO) scheme.

A more straightforward and efficient approach for high-resolution compressible flow simulation is to use the hybrid scheme. The basic idea of hybrid schemes is to use low dissipation schemes such as compact schemes in smooth regions and switch to shock-capturing schemes around discontinuities. Adam and Shariff [23] proposed a hybrid compact-ENO scheme, which shows good performance in both regions. Following a similar procedure, Pirozzoli [24] developed a hybrid compact-WENO scheme. Instead of switching from the compact scheme to the WENO scheme abruptly, Ren et al. [25] developed a characteristic-wise hybrid compact-WENO scheme, which is a weighted combination of these two schemes. Hill and Pullin [26] combined the tuned central-difference scheme and the WENO scheme. Costa and Don [27] used the spectral method as the low dissipation subscheme. Cheng et al. [28] combined the Runge-Kutta Discontinuous Galerkin method with the WENO scheme. Numerical results showed that these hybrid schemes have lower dissipation for smooth solution than shock-capturing schemes and maintain the capability of resolving discontinuities without oscillations.

The most important issue of the hybrid scheme is how to detect a shock accurately and efficiently. Many functions were proposed to develop shock detecting method, such as the flow variable difference between neighboring points [23, 24, 26], the flow gradients [18, 29], or the multi-resolution coefficients $[27,30]$. Although these functions have been successfully applied to develop hybrid schemes, a common shortcoming is that empirical problem-dependent parameters are introduced as threshold to turn on/off the shock-capturing scheme, and hence, the robustness and accuracy of the corresponding hybrid scheme are greatly reduced [15, 25, 31-34].

In this paper, by applying the nonlinear weights of the WENO-Z scheme, a problem-independent weighting switch function is proposed. Based on this new switch function and the weighting combination methodology, a new hybrid scheme is developed, which increases the accuracy of the fifth-order CRWENO scheme to seventh order in smooth region. The new scheme uses the same point stencil as the fifth-order CRWENO scheme, and it is uniformly seventh-order accurate in smooth regions while maintains the same shock-capturing capability as the CRWENO scheme.

This paper is organized as follows. High-order upwind compact schemes and the CRWENO scheme are briefly introduced in Section 2 . The weighting switch function is proposed and analyzed in Section 3. In section 4.2, the hybrid methodology is introduced, and a seventh-order hybrid scheme is constructed. Numerical results and comparisons are presented in Section 5. Conclusions are given in Section 6.

\section{HIGH-ORDER UPWIND COMPACT SCHEMES}

Without loss of generality, we describe the CRWENO scheme and the seventh-order upwind compact scheme by considering the scalar conservation law given by

$$
\frac{\partial u}{\partial t}+\frac{\partial f(u)}{\partial x}=0
$$

where $u(x, t)$ is the conserved variable, and $f(u)$ is the flux function. 
On a uniform grid where $\Delta x=x_{i+1}-x_{i}$, we look for a numerical flux function $\hat{f}_{i+\frac{1}{2}}$ such that the flux difference approximates $f_{i}^{\prime}=\partial f(u) /\left.\partial x\right|_{x=x_{i}}$ to $k$-th order accuracy:

$$
\frac{\hat{f}_{i+\frac{1}{2}}-\hat{f}_{i-\frac{1}{2}}}{\Delta x}=f_{i}^{\prime}+O\left(\Delta x^{k}\right) \text {. }
$$

The numerical flux $\hat{f}_{i+\frac{1}{2}}$ can be approximated by high-order tridiagonal compact schemes expressed as follows [24]:

$$
\alpha_{-1} \hat{f}_{i-\frac{1}{2}}+\alpha_{0} \hat{f}_{i+\frac{1}{2}}+\alpha_{1} \hat{f}_{i+\frac{3}{2}}=\sum_{m=-M_{1}}^{M_{2}} \beta_{m} f\left(x_{i+m}\right) .
$$

$M_{1}$ and $M_{2}$ can be adjusted according to the desired accuracy and the size of the point stencil. On the 5-points WENO stencil $S^{5}=\left(x_{i-2}, x_{i-1}, x_{i}, x_{i+1}, x_{i+2}\right)$, the fifth $\left(C_{5}\right)$ and seventh-order $\left(C_{7}\right)$ compact schemes are obtained as follows:

$$
\begin{gathered}
C_{5}: \frac{3}{10} \hat{f}_{i-\frac{1}{2}}+\frac{6}{10} \hat{f}_{i+\frac{1}{2}}+\frac{1}{10} \hat{f}_{i+\frac{3}{2}}=\frac{1}{30} f_{i-1}+\frac{19}{30} f_{i}+\frac{10}{30} f_{i+1} \\
C_{7}: \frac{2}{7} \hat{f}_{i-\frac{1}{2}}+\frac{4}{7} \hat{f}_{i+\frac{1}{2}}+\frac{1}{7} \hat{f}_{i+\frac{3}{2}}=-\frac{1}{420} f_{i-2}+\frac{19}{420} f_{i-1}+\frac{239}{420} f_{i}+\frac{53}{140} f_{i+1}+\frac{1}{105} f_{i+2} .
\end{gathered}
$$

Eq. (4) can be expressed as a convex combination of three third-order compact schemes:

$$
\begin{aligned}
& C_{3}^{0}: \frac{2}{3} \hat{f}_{i-\frac{1}{2}}+\frac{1}{3} \hat{f}_{i+\frac{1}{2}}=\frac{1}{6} f_{i-1}+\frac{5}{6} f_{i} \\
& C_{3}^{1}: \frac{1}{3} \hat{f}_{i-\frac{1}{2}}+\frac{2}{3} \hat{f}_{i+\frac{1}{2}}=\frac{5}{6} f_{i}+\frac{1}{6} f_{i+1} \\
& C_{3}^{2}: \frac{2}{3} \hat{f}_{i+\frac{1}{2}}+\frac{1}{3} \hat{f}_{i+\frac{3}{2}}=\frac{1}{6} f_{i}+\frac{5}{6} f_{i+1}
\end{aligned}
$$

with coefficients $c_{0}=\frac{2}{10}, c_{1}=\frac{5}{10}$, and $c_{2}=\frac{3}{10}$, respectively.

By replacing $c_{k}$ with nonlinear WENO weights, the CRWENO scheme [22] is constructed as follows:

$$
C_{\mathrm{CRWENO} 5}: \tilde{\alpha}_{-1}^{5} \hat{f}_{i-\frac{1}{2}}+\tilde{\alpha}_{0}^{5} \hat{f}_{i+\frac{1}{2}}+\tilde{\alpha}_{1}^{5} \hat{f}_{i+\frac{3}{2}}=\sum_{r=-1}^{1} \tilde{\beta}_{r}^{5} f_{i+r}
$$

in which

$$
\begin{aligned}
& \tilde{\alpha}_{-1}^{5}=\frac{2}{3} \omega_{0}+\frac{1}{3} \omega_{1}, \quad \tilde{\alpha}_{0}^{5}=\frac{1}{3} \omega_{0}+\frac{2}{3}\left(\omega_{1}+\omega_{2}\right), \quad \tilde{\alpha}_{1}^{5}=\frac{1}{3} \omega_{2}, \\
& \tilde{\beta}_{-1}^{5}=\frac{\omega_{0}}{6}, \quad \tilde{\beta}_{0}^{5}=\frac{5\left(\omega_{0}+\omega_{1}\right)+\omega_{2}}{6}, \quad \tilde{\beta}_{1}^{5}=\frac{\omega_{1}+5 \omega_{2}}{6},
\end{aligned}
$$

and the nonlinear weights $\omega_{k}$ are defined as follows:

$$
\omega_{k}=\frac{a_{k}}{\sum_{i} a_{i}}, a_{k}=\frac{c_{k}}{\left(\varepsilon+\beta_{k}\right)^{p}}, k=\{0,1,2\}, p=1,2, \ldots
$$

$\beta_{k}$ are the smoothness indicators given by [2]

$$
\beta_{0}=\frac{13}{12}\left(f_{i-2}-2 f_{i-1}+f_{i}\right)^{2}+\frac{1}{4}\left(f_{i-2}-4 f_{i-1}+3 f_{i}\right)^{2}
$$




$$
\begin{gathered}
\beta_{1}=\frac{13}{12}\left(f_{i-1}-2 f_{i}+f_{i+1}\right)^{2}+\frac{1}{4}\left(f_{i-1}-f_{i+1}\right)^{2} \\
\beta_{2}=\frac{13}{12}\left(f_{i}-2 f_{i+1}+f_{i+2}\right)^{2}+\frac{1}{4}\left(3 f_{i}-4 f_{i+1}+f_{i+2}\right)^{2} .
\end{gathered}
$$

$\epsilon$ is a small number to avoid division by zero.

As analyzed by Henrick et al. [3], for the fifth-order WENO scheme, nonlinear weights (10) do not satisfy the necessary and sufficient conditions for fifth-order convergence; a mapping function was introduced to improve the accuracy of the final weights (the WENO-M scheme). In [4], Borges et al. introduced a parameter

$$
\tau_{5}=\left|\beta_{0}-\beta_{2}\right|
$$

to calculate the weights as follows:

$$
a_{k}=c_{k}\left(1+\left(\frac{\tau_{5}}{\beta_{k}+\varepsilon}\right)^{q}\right), k=\{0,1,2\}, q=1,2, \ldots
$$

This new scheme (the WENO-Z scheme) is less computational expensive than the WENO-M scheme.

\section{THE NEW WEIGHTING SWITCH FUNCTION}

The switch method determines the performance of hybrid schemes. As mentioned previously, most switch methods employ empirical problem-dependent parameters to turn on the shock-capturing scheme. These parameters greatly limits the application range of hybrid schemes.

By using $\alpha_{k}$ given in (15), a high-order problem-independent weighting switch function is proposed as the following:

$$
\theta=\frac{1}{1+\left(\sum_{k} a_{k}-1\right)^{2}}
$$

To evaluate the behavior of $\theta$ at both smooth and discontinuous stencils, let us recall the main features of $\tau_{5}$ given in (14) and summarized in [4]:

- If the WENO stencil $S^{5}=\left(x_{i-2}, x_{i-1}, x_{i}, x_{i+1}, x_{i+2}\right)$ does not contain discontinuities, then, $\tau_{5}=O\left(\Delta x^{5}\right) \ll \beta_{k}$ for $k=0,1,2$;

- if the solution is continuous at some of the $S_{k}=\left(x_{i-2+k}, x_{i-1+k}, x_{i+k}\right), k=0,1,2$, but discontinuous in the whole $S_{5}$, then, $\beta_{k} \ll \tau_{5}$, for those $k$ where the solution is continuous;

- $\tau_{5} \leqslant \max \beta_{k}, k=0,1,2$.

Therefore, for those stencils containing discontinuities, we have

$$
\sum_{k} a_{k} \gg 1
$$

and thus,

$$
\theta \approx 0
$$

For smooth stencils, performing Taylor expansion to $f_{i+k}=f\left(x_{i}+k \Delta x\right)$ in the smoothness indicators $\beta_{k}$ and $\tau_{5}$ around $x_{i}$ : 


$$
\begin{gathered}
\beta_{0}=f_{i}^{\prime 2} \Delta x^{2}+\left(\frac{13}{12} f_{i}^{\prime \prime 2}-\frac{2}{3} f_{i}^{\prime} f_{i}^{\prime \prime \prime}\right) \Delta x^{4} \\
+\left(-\frac{13}{6} f_{i}^{\prime \prime} f_{i}^{\prime \prime \prime}+\frac{1}{2} f_{i}^{\prime} f_{i}^{\prime \prime \prime \prime}\right) \Delta x^{5}+O\left(\Delta x^{6}\right) \\
\beta_{1}=f_{i}^{\prime 2} \Delta x^{2}+\left(\frac{13}{12} f_{i}^{\prime \prime 2}-\frac{2}{3} f_{i}^{\prime} f_{i}^{\prime \prime \prime}\right) \Delta x^{4}+O\left(\Delta x^{6}\right), \\
\beta_{2}=f_{i}^{\prime 2} \Delta x^{2}+\left(\frac{13}{12} f_{i}^{\prime \prime 2}-\frac{2}{3} f_{i}^{\prime} f_{i}^{\prime \prime \prime}\right) \Delta x^{4} \\
+\left(\frac{13}{6} f_{i}^{\prime \prime} f_{i}^{\prime \prime \prime}-\frac{1}{2} f_{i}^{\prime} f_{i}^{\prime \prime \prime \prime}\right) \Delta x^{5}+O\left(\Delta x^{6}\right) \\
\tau_{5}=\left|\frac{13}{3} f_{i}^{\prime \prime} f_{i}^{\prime \prime \prime}-f_{i}^{\prime} f_{i}^{(4)}\right| \Delta x^{5}+O\left(\Delta x^{6}\right)
\end{gathered}
$$

and

$$
a_{k}=c_{k}\left(1+\left(\frac{\tau_{5}}{\beta_{k}+\epsilon}\right)^{q}\right)= \begin{cases}c_{k}+O\left(\Delta x^{3 q}\right), & f^{\prime} \neq 0 \\ c_{k}+O\left(\Delta x^{q}\right), & f^{\prime}=0\end{cases}
$$

we obtain the truncation error of $\theta$ :

$$
\theta=\frac{1}{1+\left(\sum_{k} a_{k}-1\right)^{2}}=1-O\left(\Delta x^{p}\right), \quad p= \begin{cases}6 q, & f^{\prime} \neq 0 \\ 2 q, & f^{\prime}=0\end{cases}
$$

Eq. (22) shows that $\theta$ is a high-order approximation of 1 at smooth stencils. Clearly,

$$
0<\theta<1
$$

In fact, the switch function $\theta$ can be constructed in a general form:

$$
\theta(x)=\frac{1}{1+x^{z}}, \quad x=\sum_{k} a_{k}-1 .
$$

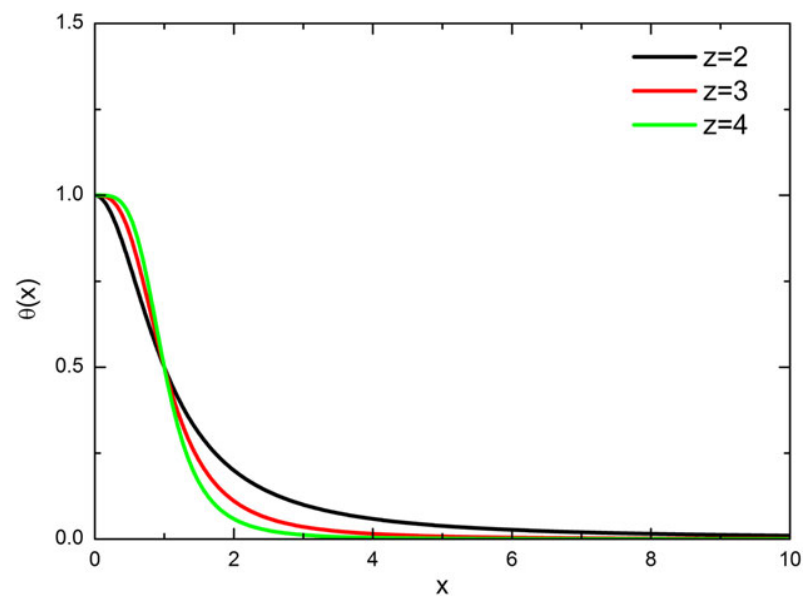

Figure 1. $\theta(x)$ with different $z$ values. [Colour figure can be viewed at wileyonlinelibrary.com] 
Figure 1 plots the profile of $\theta$ versus $x$. It can be observed that, with larger $z, \theta$ converges to 1 faster. This property makes $\theta$ flexible in applications. This will be discussed in the next section.

\section{DEVELOPING HYBRID SCHEMES WITH $\theta$ FUNCTION}

\subsection{Hybrid method}

Based on $\theta$ function, we apply the following method to hybridize a linear scheme and a WENO scheme :

$$
F_{\text {Hybrid }}=(1-\theta) F_{W E N O}+\theta F_{\text {Linear }}
$$

in which $F_{W E N O}$ stands for the formula of a certain WENO scheme (e.g., WENO-Z or CRWENO), and $F_{\text {Linear }}$ stands for a certain high-order linear scheme. For smooth regions, as $\theta \approx 1, F_{\text {Hybrid }}$ approaches $F_{\text {Linear }}$. For discontinuities, $\theta \approx 0$ and, therefore, $F_{H y b r i d}$ approach $F_{W E N O}$. The designed behavior of $F_{H y b r i d}$ around discontinuities indicates that the shock capability of hybrid schemes developed with (24) is the same as the underlying WENO scheme.

In the following part, as an example, a seventh-order hybrid scheme is constructed with the proposed method (24), and the accuracy of the new scheme is also analyzed.

\subsection{The hybrid compact-CRWENO scheme}

It has been shown that the fifth-order CRWENO scheme is more accurate than the fifth-order WENO schemes $[22,35]$. Utilizing the same stencil size and the same left hand side structure of the seventhorder upwind compact scheme (5) and the CRWENO (9), we construct the new hybrid compactCRWENO scheme as the following:

$$
C_{H C C S}:(1-\theta) C_{C R W E N O 5}+\theta C_{7},
$$

that is,

$$
C_{H C C S}: \tilde{\alpha}_{-1} \hat{f}_{i-\frac{1}{2}}+\tilde{\alpha}_{0} \hat{f}_{i+\frac{1}{2}}+\tilde{\alpha}_{1} \hat{f}_{i+\frac{3}{2}}=\sum_{m=-2}^{2} \tilde{\beta}_{m} f_{i+m},
$$

where $\tilde{\alpha}_{k}, \tilde{\beta}_{k}$ are given by

$$
\begin{aligned}
\tilde{\alpha}_{-1} & =\frac{2}{7} \theta+(1-\theta)\left(\frac{2}{3} \omega_{0}+\frac{1}{3} \omega_{1}\right), \\
\tilde{\alpha}_{0} & =\frac{4}{7} \theta+(1-\theta)\left(\frac{1}{3} \omega_{0}+\frac{2}{3}\left(\omega_{1}+\omega_{2}\right)\right), \\
\tilde{\alpha}_{1} & =\frac{1}{7} \theta+(1-\theta) \frac{1}{3} \omega_{2}, \\
\tilde{\beta}_{-2} & =-\frac{1}{420} \theta \\
\tilde{\beta}_{-1} & =\frac{19}{420} \theta+(1-\theta) \frac{\omega_{0}}{6} \\
\tilde{\beta}_{0} & =\frac{239}{420} \theta+(1-\theta) \frac{5\left(\omega_{0}+\omega_{1}\right)+\omega_{2}}{6}, \\
\tilde{\beta}_{1} & =\frac{53}{140} \theta+(1-\theta) \frac{\omega_{1}+5 \omega_{2}}{6}, \\
\tilde{\beta}_{2} & =\frac{1}{105} \theta .
\end{aligned}
$$


$\omega_{k}$ are the WENO-Z weights, and $\theta$ is obtained with (16). Here, it should be pointed out that formula (24) is an explicit weighted combination of different flux functions, while (25) is an implicit one, because compact schemes are used.

To analyze the property of the new hybrid scheme, we rewrite (26) as

$$
(1-\theta) C_{5}+(1-\theta) \sum_{k}\left(\omega_{k}-c_{k}\right) C_{3}^{k}+\theta C_{7}
$$

Obviously, for non-smooth stencils, as $\theta \approx 0$, the new hybrid scheme approaches the CRWENO scheme.

For smooth stencils, we have $\theta \approx 1$, and thus, HCCS approaches the seventh-order compact scheme. Note that, for an $n$-th order compact scheme,

$$
C_{n}: C_{n, L}\left(\ldots, f_{i-1 / 2}, f_{i+1 / 2}, f_{i+3 / 2}, \ldots\right)=C_{n, R}\left(\ldots, f_{i-1}, f_{i}, f_{i+1}, \ldots\right) .
$$

The right hand side of (28) is an $n$-th order approximation of the left hand side [24]:

$$
C_{n, L}-C_{n, R}=O\left(\Delta x^{n}\right) .
$$

For example, for $C_{5}$ (Eq. (4)), we have

$$
C_{5, L}-C_{5, R}=\frac{3}{10} \hat{f}_{i-\frac{1}{2}}+\frac{6}{10} \hat{f}_{i+\frac{1}{2}}+\frac{1}{10} \hat{f}_{i+\frac{3}{2}}-\left(\frac{1}{30} f_{i-1}+\frac{19}{30} f_{i}+\frac{10}{30} f_{i+1}\right) \approx O\left(\Delta x^{5}\right) .
$$

Therefore, (27) can be then written as follows:

$$
\begin{aligned}
C_{H C C S, L}-C_{H C C S, R}= & (1-\theta) O_{5}\left(\Delta x^{5}\right)+(1-\theta) \sum_{k=0}^{2}\left(\omega_{k}-c_{k}\right) O_{3}^{k}\left(\Delta x^{3}\right) \\
& +\theta O_{7}\left(\Delta x^{7}\right) .
\end{aligned}
$$

If the leading order of the right hand side of (29) is seventh order, the new hybrid scheme is seventhorder accurate for smooth stencils. Therefore, a sufficient condition for seventh-order convergence of HCCS can be obtained as follows:

$$
\left\{\begin{array}{l}
1-\theta=O\left(\Delta x^{2}\right) \\
(1-\theta)\left(\omega_{k}-c_{k}\right)=O\left(\Delta x^{4}\right) .
\end{array}\right.
$$

Furthermore, given that $1-\theta=O\left(\Delta x^{p}\right)$ (Eq. (22)) and $\omega_{k}-c_{k}=O\left(\Delta x^{s}\right)$, the sufficient condition becomes

$$
\left\{\begin{array}{l}
p \geqslant 2 \\
p+s \geqslant 4
\end{array}\right.
$$

Consequently, if $q=2$ is taken to calculate $\alpha_{k}$ in Eq. (15), the new hybrid scheme is always seventh-order accurate in smooth regions, even at critical points where $s=1$.

It is worth noting that the aforementioned analysis still holds if one employs the general form of $\theta$ (23) with different $z$ to combine WENO schemes with other linear schemes.

\section{NUMERICAL TESTS}

In this section, several numerical tests including scalar, one dimensional and two dimensional problems are considered to evaluate the performances of HCCS. To show that the proposed switch function is problem independent, numerical results of HCCS for scalar problems are compared with those of the hybrid scheme proposed in [24]. Results of HCCS are compared with the CRWENO scheme, the WENO-Z scheme, and the seventh-order WENO scheme $[12,36]$ for all problems to show numerical properties of the present hybrid method. 
The explicit third-order total variation diminishing Runge-Kutta method [37] is used for time marching:

$$
\begin{gathered}
u^{(1)}=u^{n}+\Delta t L\left(u^{n}\right) \\
u^{(2)}=\frac{3}{4} u^{n}++\frac{1}{4} u^{(1)}+\frac{1}{4} \Delta t L\left(u^{(1)}\right) \\
u^{n+1}=\frac{1}{3} u^{n}+\frac{2}{3} u^{(2)}+\frac{2}{3} \Delta t L\left(u^{(2)}\right) .
\end{gathered}
$$

For one dimensional Euler's equations with strong discontinuities, characteristic-wise reconstruction, which reconstructs the eigenvectors of the Euler flux [2], is used as in [22, 25]. For two dimensional problems, the Steger-Warming flux splitting method [38] is applied. The value of $\varepsilon$ may influence the result of numerical simulations [9]. $\varepsilon$ is set to $1 \times 10^{-40}$ for linear equation tests and $1 \times 10^{-6}$ for remainder of the tests.

\subsection{Linear advection equation}

The linear advection equation is given by

$$
\left\{\begin{array}{l}
u_{t}+u_{x}=0, \quad-1 \leqslant x \leqslant 1 \\
u(x, 0)=u_{0}(x), \quad \text { periodic boundary. }
\end{array}\right.
$$

The exact solution of (35) at time $t$ with the initial condition $u_{0}(x)$ is given by

$$
u(x, t)=u_{0}(x-t) .
$$
by

Two initial conditions are considered in this section. The initial condition of the first case is given

$$
u_{0}(x)=\sin \left(\pi x-\frac{\sin \pi x}{\pi}\right) .
$$

This initial condition has two critical points, where $f^{\prime}=0$ and $f^{\prime \prime \prime} \neq 0$ [3]. The time step $\Delta t$ is set to $\Delta x^{n / 3}$, where $n$ is the order of the scheme being studied.

Table I gives the errors and accuracy orders of different schemes with different grid number $N$. The $L_{2}$ norm of the error is obtained by comparison with the exact solution at $t=2$ according to

\begin{tabular}{|c|c|c|c|c|c|c|c|c|}
\hline \multirow[b]{2}{*}{$N$} & \multicolumn{2}{|c|}{ WENO-Z } & \multicolumn{2}{|c|}{ CRWENO } & \multicolumn{2}{|c|}{ WENO-7 } & \multicolumn{2}{|c|}{ HCCS } \\
\hline & $L_{2}$ & order & $L_{2}$ & order & $L_{2}$ & order & $L_{2}$ & order \\
\hline 20 & $3.92 \mathrm{E}-03$ & - & $1.88 \mathrm{E}-03$ & - & $6.05 \mathrm{E}-04$ & - & $1.09 \mathrm{E}-03$ & - \\
\hline 40 & $1.38 \mathrm{E}-04$ & 4.83 & $3.14 \mathrm{E}-05$ & 5.90 & $5.10 \mathrm{E}-06$ & 6.89 & $1.85 \mathrm{E}-06$ & 9.23 \\
\hline 80 & 4.39E-06 & 4.97 & $7.20 \mathrm{E}-07$ & 5.48 & $4.16 \mathrm{E}-08$ & 6.94 & 8.68E-09 & 7.76 \\
\hline 160 & $1.37 \mathrm{E}-07$ & 5.00 & $2.47 \mathrm{E}-08$ & 4.86 & $3.29 \mathrm{E}-10$ & 6.98 & $8.92 \mathrm{E}-11$ & 6.62 \\
\hline 320 & $4.28 \mathrm{E}-09$ & 5.00 & $8.40 \mathrm{E}-10$ & 4.88 & $2.58 \mathrm{E}-12$ & 7.00 & $7.97 \mathrm{E}-13$ & 6.83 \\
\hline
\end{tabular}

$$
L_{2}=\sqrt{\frac{1}{N} \sum_{i=1}^{N}\left(u_{i}-u_{\text {exact }, i}\right)^{2}} .
$$

The seventh-order WENO scheme is used to calculate the fluxes at boundaries for HCCS. It can be seen that HCCS achieves near seventh order, and the errors are three magnitudes small than the

Table I. $L_{2}$ errors and accuracy orders of different schemes for the initial condition (37)

$$
\text { at } t=2 \text {. }
$$


base CRWENO scheme. HCCS is also more accurate than the seventh-order WENO scheme for this smooth problem.

In the case with very coarse mesh, for example, $N=20$, the switch function is affected by the mesh size severely, and hence, the error of HCCS approaches to CRWENO and WENO schemes. But with refined mesh $(N=40)$, the error of HCCS approaches to the seventh-order compact scheme; therefore, Table I shows super-convergence(convergence order larger than ideal order)[2]. As grid number further increased, the accuracy order recovery about seventh order.

Different boundary formulas are tested for the first case. Table II compares the errors of HCCS by using the sixth-order and eighth-order central schemes as boundary formula. It can be seen that, with the sixth-order formula, HCCS converges at sixth order. With eighth-order formula, HCCS achieves seventh order, and its errors are less than those with WENO-7 as boundary formula. Hence, for other cases in this paper, the eighth-order central scheme is applied to calculate the fluxes at boundaries.

The influence of the parameter $z$ in (23) on the accuracy of HCCS is also studied. The comparison given in Table III shows that, with $z=1$, the errors are almost an order of magnitude larger than other cases. For $z=2,3,4$, the differences are mainly on coarse meshes. Therefore, to achieve seventh-order convergence, the value of $z$ can be arbitrarily chosen as long as $z \geqslant 2$. Larger $z$ only has negligible effect on computation efficiency and accuracy.

CPU times per time step of different schemes are compared in Table IV. HCCS is about $20 \%$ faster than the seventh-order WENO scheme. Figure 2 shows CPU time versus error for different schemes. It can be seen that to obtain the same level of error, HCCS requires the least CPU time, which indicates that HCCS is more efficient than the other schemes.

Table II. $L_{2}$ errors of HCCS with the sixth and eighth order central schemes at boundaries.

\begin{tabular}{lccccc}
\hline & \multicolumn{2}{c}{ Sixth order } & & \multicolumn{2}{c}{ Eighth order } \\
\cline { 2 - 3 } \cline { 5 - 5 }$N$ & $L_{2}$ & order & & $L_{2}$ & order \\
\hline 20 & $1.05 \mathrm{E}-03$ & - & & $1.06 \mathrm{E}-03$ & - \\
40 & $7.38 \mathrm{E}-06$ & 7.15 & & $1.68 \mathrm{E}-06$ & 9.32 \\
80 & $1.75 \mathrm{E}-07$ & 5.41 & & $3.92 \mathrm{E}-09$ & 8.74 \\
160 & $3.63 \mathrm{E}-09$ & 5.61 & & $2.76 \mathrm{E}-11$ & 7.15 \\
320 & $6.46 \mathrm{E}-11$ & 5.83 & & $1.96 \mathrm{E}-13$ & 7.14 \\
\hline
\end{tabular}

Table III. Accuracy for hybrid compact-CRWENO scheme with different value of $z$.

\begin{tabular}{|c|c|c|c|c|c|c|c|c|}
\hline \multirow[b]{2}{*}{$N$} & \multicolumn{2}{|c|}{$z=1$} & \multicolumn{2}{|c|}{$z=2$} & \multicolumn{2}{|c|}{$z=3$} & \multicolumn{2}{|c|}{$z=4$} \\
\hline & $L_{2}$ & order & $L_{2}$ & order & $L_{2}$ & order & $L_{2}$ & order \\
\hline 20 & $9.79 \mathrm{E}-04$ & - & $1.06 \mathrm{E}-03$ & - & $1.15 \mathrm{E}-03$ & - & $1.23 \mathrm{E}-03$ & - \\
\hline 40 & $5.26 \mathrm{E}-06$ & 7.56 & $1.68 \mathrm{E}-06$ & 9.32 & $6.49 \mathrm{E}-07$ & 10.83 & 4.70E-07 & 14.72 \\
\hline 80 & 2.47E-08 & 7.76 & 3.92E-09 & 8.74 & 3.61E-09 & 7.52 & 3.61E-09 & 7.02 \\
\hline 160 & $2.91 \mathrm{E}-10$ & 6.43 & $2.76 \mathrm{E}-11$ & 7.15 & $2.72 \mathrm{E}-11$ & 7.05 & $2.72 \mathrm{E}-11$ & 7.05 \\
\hline 320 & $3.14 \mathrm{E}-12$ & 6.56 & $1.96 \mathrm{E}-13$ & 7.14 & $1.95 \mathrm{E}-13$ & 7.12 & $1.95 \mathrm{E}-13$ & 7.12 \\
\hline
\end{tabular}

Table IV. Averaged CPU times (in seconds) per time step for different schemes.

\begin{tabular}{lcccc}
\hline$N$ & WENO-Z & CRWENO & WENO-7 & HCCS \\
\hline 20 & $3.36 \mathrm{E}-04$ & $3.83 \mathrm{E}-04$ & $5.21 \mathrm{E}-04$ & $4.34 \mathrm{E}-04$ \\
40 & $5.15 \mathrm{E}-04$ & $6.60 \mathrm{E}-04$ & $9.45 \mathrm{E}-04$ & $7.38 \mathrm{E}-04$ \\
80 & $1.09 \mathrm{E}-03$ & $1.30 \mathrm{E}-03$ & $1.86 \mathrm{E}-03$ & $1.48 \mathrm{E}-03$ \\
160 & $2.09 \mathrm{E}-03$ & $2.58 \mathrm{E}-03$ & $3.73 \mathrm{E}-03$ & $2.91 \mathrm{E}-03$ \\
320 & $4.22 \mathrm{E}-03$ & $5.10 \mathrm{E}-03$ & $7.27 \mathrm{E}-03$ & $5.71 \mathrm{E}-03$ \\
640 & $8.41 \mathrm{E}-03$ & $1.02 \mathrm{E}-02$ & $1.40 \mathrm{E}-02$ & $1.13 \mathrm{E}-02$ \\
\hline
\end{tabular}




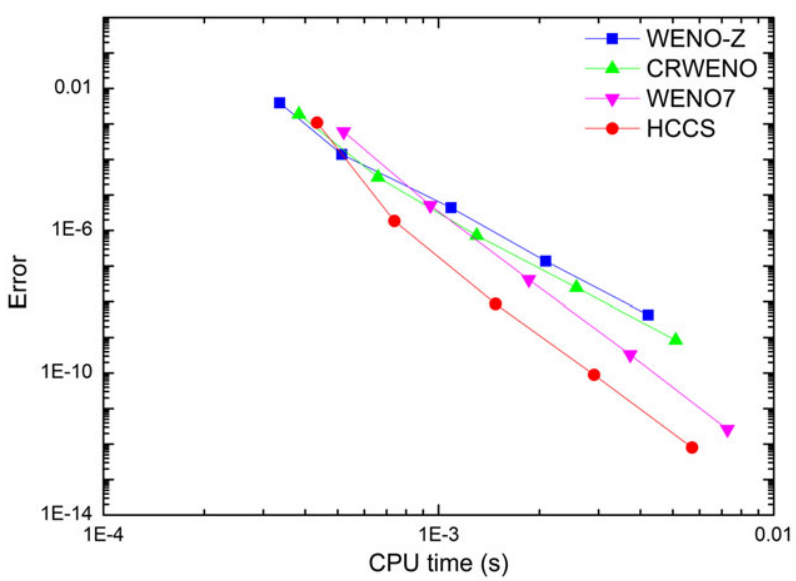

Figure 2. CPU time versus Error. [Colour figure can be viewed at wileyonlinelibrary.com]

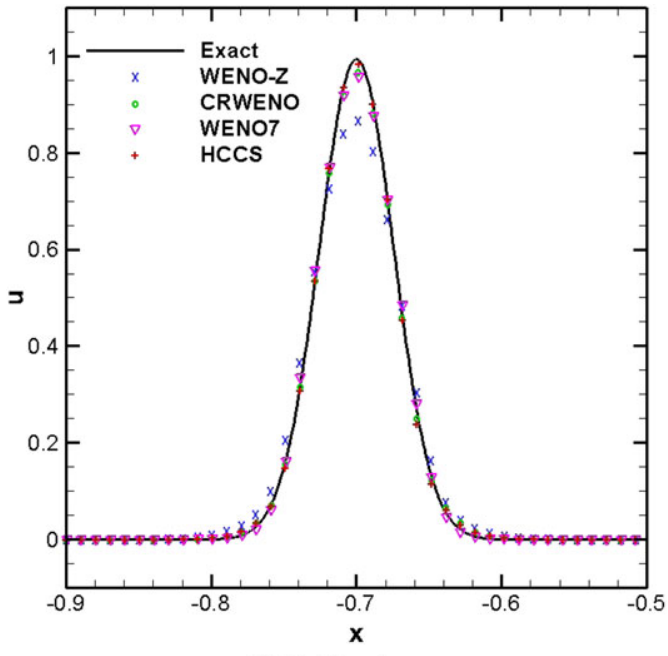

(a) The Gaussian wave

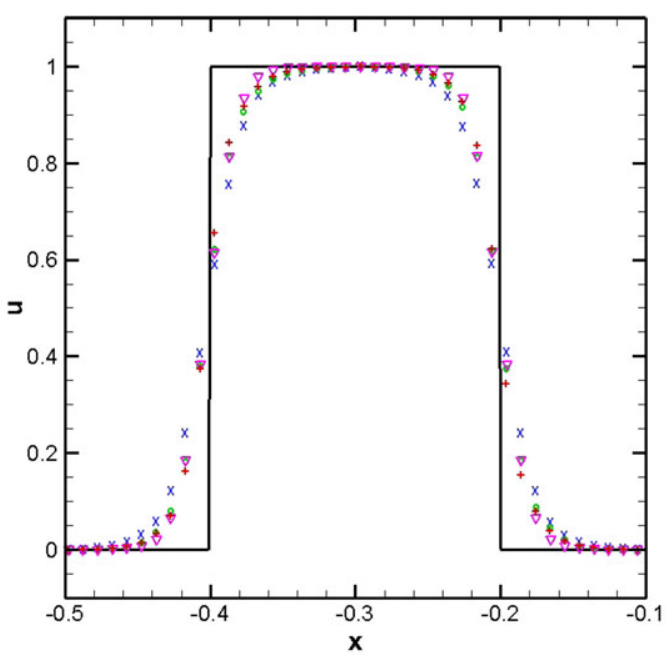

(b) The square wave

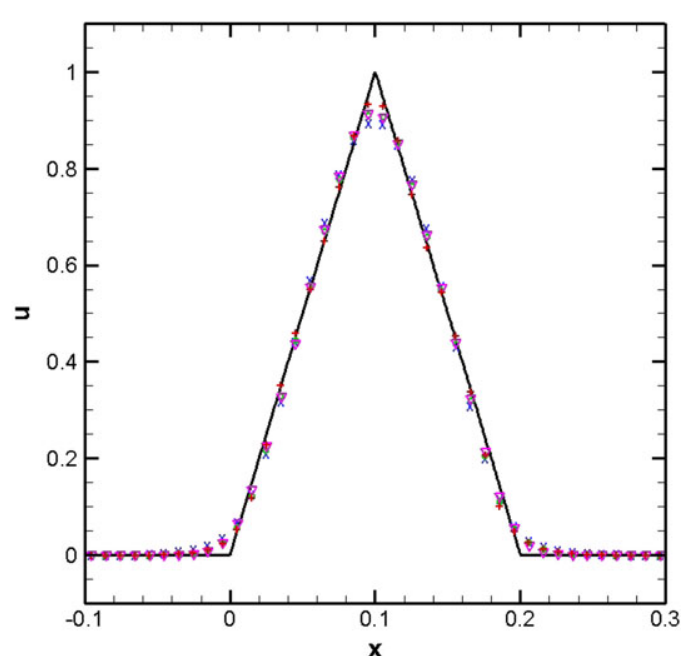

(c) The triangle wave

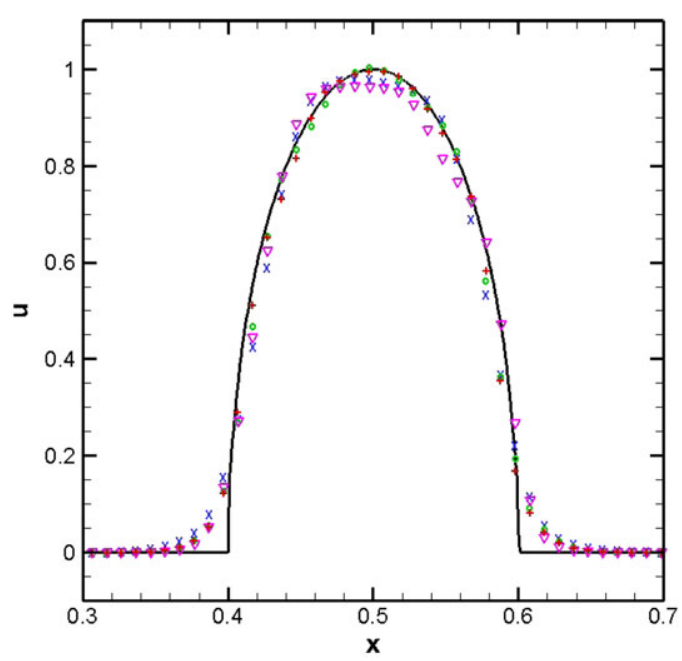

(d) The ellipse wave

Figure 3. Results of the linear advection equation with the initial condition (37) at $t=6, N=200$. [Colour figure can be viewed at wileyonlinelibrary.com] 
The initial condition of the second case is

$$
u(x, 0)=\left\{\begin{array}{lc}
\frac{1}{6}(G(x, \beta, z-\delta)+G(x, \beta, z+\delta)+4 G(x, \beta, z)), & -0.8 \leqslant x<-0.6 \\
1, & -0.4 \leqslant x<-0.2 \\
1-|10(x-0.1)|, & 0 \leqslant x<0.2 \\
\frac{1}{6}(F(x, \alpha, a-\delta)+F(x, \alpha, a+\delta)+4 F(x, \alpha, a)), & 0.4 \leqslant x<0.6 \\
0, & \text { otherwise }
\end{array}\right.
$$

Table V. $L_{2}$ errors and accuracy orders of HS-P and HCCS for the initial condition (37) at $\mathrm{t}=2$.

\begin{tabular}{lcccccccc}
\hline & \multicolumn{2}{c}{ HS-P $\beta=0.01$} & & \multicolumn{2}{c}{ HS-P $\beta=0.8$} & & \multicolumn{2}{c}{ HCCS } \\
\cline { 2 - 3 } $\mathrm{N}$ & $L_{2}$ & order & & $L_{2}$ & order & & $L_{2}$ & order \\
\hline 20 & $3.62 \mathrm{E}-03$ & - & & $1.06 \mathrm{E}-03$ & - & & $1.06 \mathrm{E}-03$ & - \\
40 & $2.24 \mathrm{E}-04$ & 4.01 & & $3.16 \mathrm{E}-05$ & 5.07 & & $1.68 \mathrm{E}-06$ & 9.32 \\
80 & $6.43 \mathrm{E}-06$ & 5.12 & & $9.70 \mathrm{E}-07$ & 5.02 & & $3.92 \mathrm{E}-09$ & 8.74 \\
160 & $4.09 \mathrm{E}-07$ & 3.97 & & $2.95 \mathrm{E}-08$ & 5.04 & & $2.76 \mathrm{E}-11$ & 7.15 \\
320 & $1.47 \mathrm{E}-08$ & 4.80 & & $8.81 \mathrm{E}-10$ & 5.06 & & $1.96 \mathrm{E}-13$ & 7.14 \\
\hline
\end{tabular}

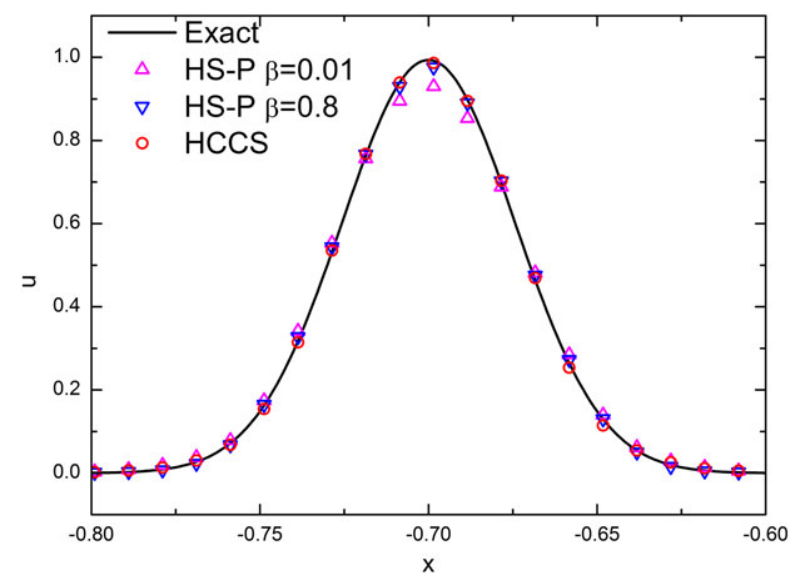

(a) The Gaussian wave

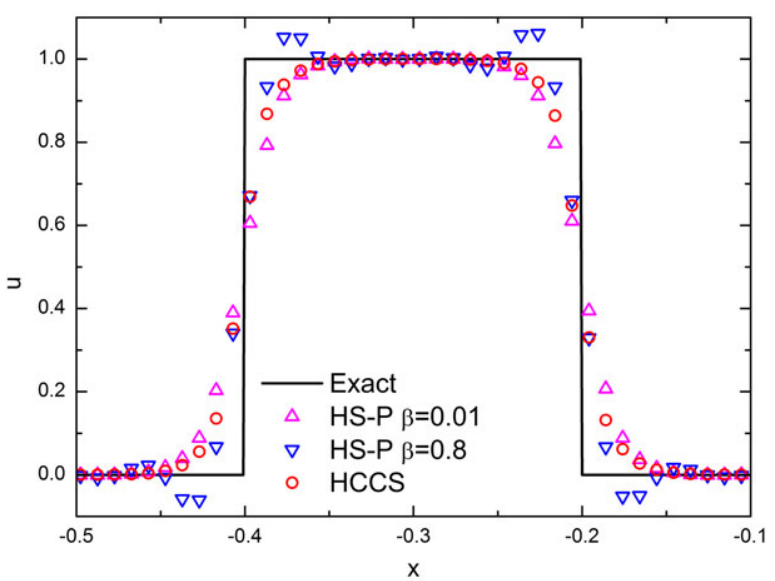

(b) The square wave

Figure 4. Comparison of HS-P and HCCS for the solutions of the Gaussian wave and the square wave. HS-P, hybrid scheme proposed; HCCS, hybrid compact-CRWENO scheme.[Colour figure can be viewed at wileyonlinelibrary.com] 
where

$$
\begin{aligned}
& G(x, \beta, z)=e^{-\beta(x-z)^{2}}, F(x, \alpha, a)=\sqrt{\max \left(1-\alpha^{2}(x-a)^{2}, 0\right)}, \\
& a=0.5, z=-0.7, \delta=0.005, \alpha=10, \beta=\log 2 / 36 \delta^{2} .
\end{aligned}
$$

The solution of (38) is consist of a smooth but narrow combination of Gaussians, a square wave, a sharp triangle wave, and a half ellipse [2]. This is a widely used benchmark to test the shockcapturing capability as well as the steep smooth wave resolution of a scheme. Results at $t=6$ of different schemes are shown in Figure 3. It can be seen that WENO-7 resolves discontinuities better than other schemes because of its wide discontinuity detecting point stencil ( 7 points are used to detect discontinuities). However, HCCS maintains the essentially non-oscillation property of CRWENO and obtains more accurate solution for smooth waves, that is, the Gaussian, the triangle, and the ellipse waves.

To illustrate the advantage of the new switch function over traditional method, we compare HCCS with the hybrid scheme proposed in [24] (referred as HS-P) for the aforementioned two linear problems. Two different values for the threshold $\beta$ are considered 0.01 and 0.8 . Table $\mathrm{V}$ gives the convergence order of HCCS and HS-P for problem (37). Figure 4 shows the square and the Gaus-

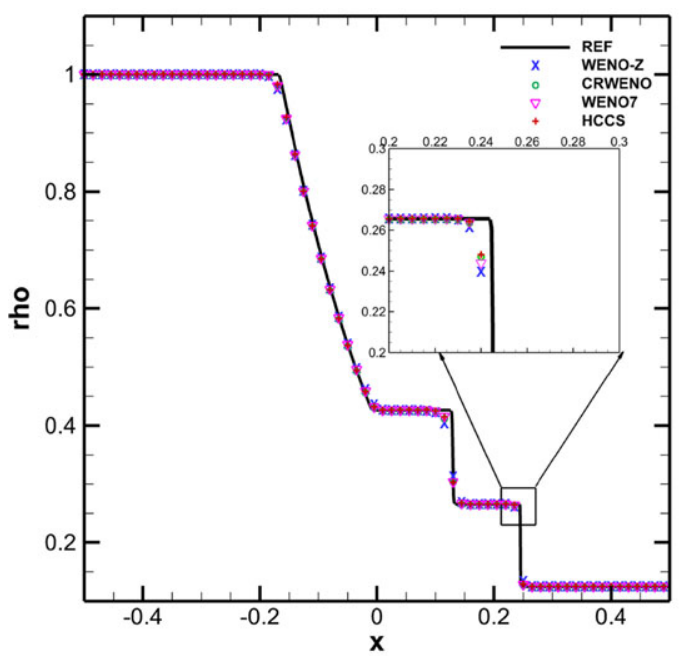

(a) The Sod problem at $\mathrm{t}=0.14$

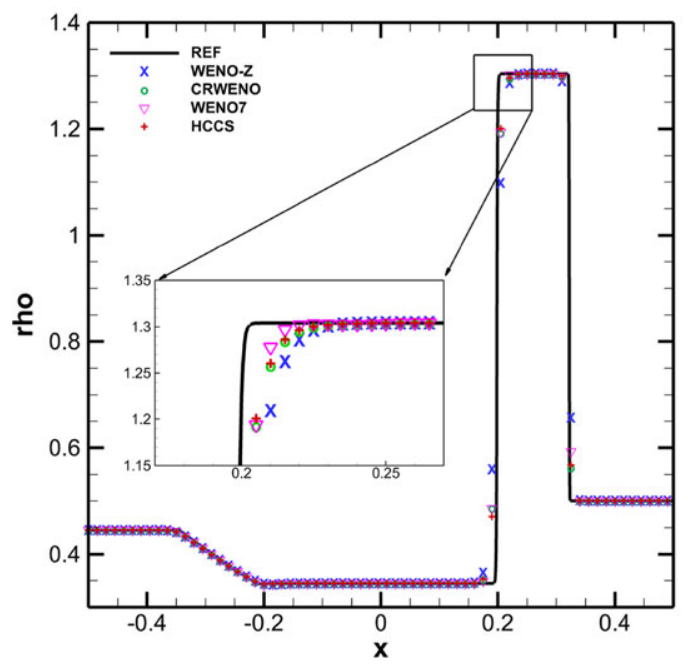

(b) The Lax problem at $\mathrm{t}=0.13$

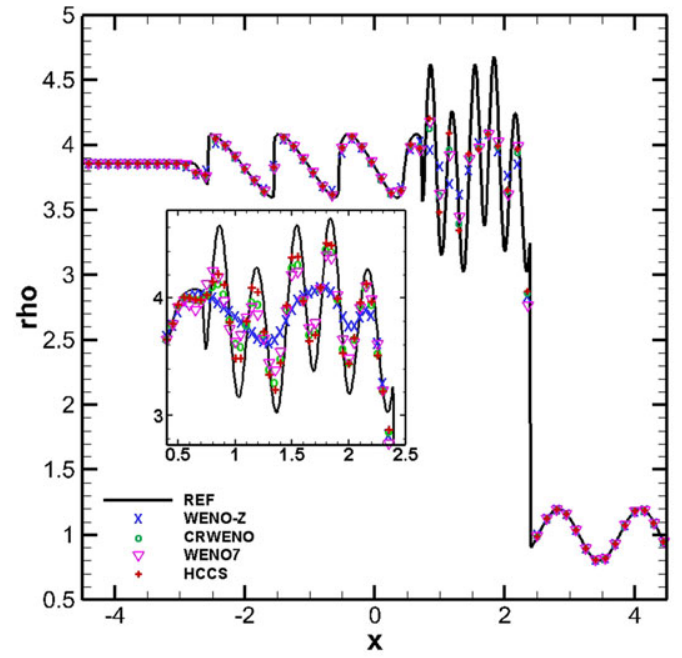

(c) The Shu-Osher problem at $t=1.8$

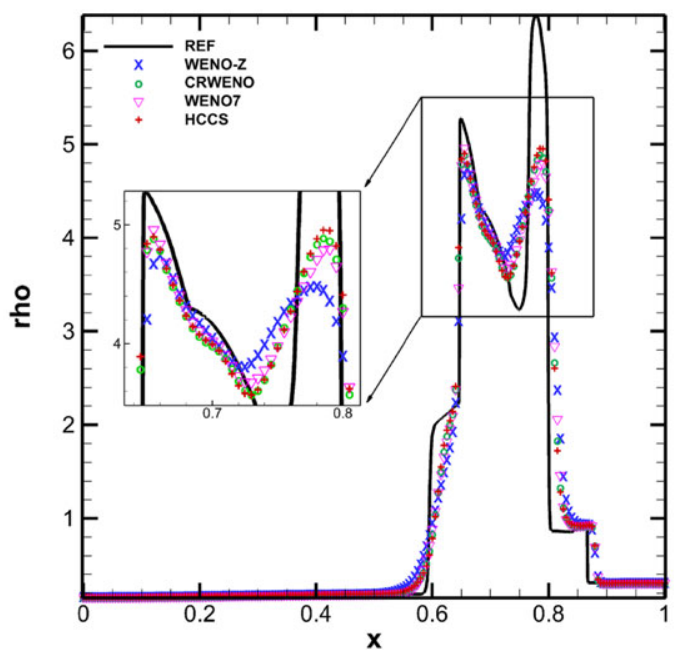

(d) The interacting blast waves problem at $\mathrm{t}=0.038$

Figure 5. Density distributions of solutions of the different $1 \mathrm{~d}$ Euler problems, one of every three points is displayed. [Colour figure can be viewed at wileyonlinelibrary.com] 
sian wave results of both schemes for initial condition (38). It can be seen that larger $\beta$ of HS-P gives more accurate solution in smooth regions but generates oscillation near discontinuities and otherwise for smaller $\beta$. That means, for a complex problem, it is difficult to achieve a good solution without the knowledge of how to select the problem-dependent parameter[31]. However, HCCS is free of such parameter tuning process and gives accurate and non-oscilatory results for both smooth and discontinous solutions.

\subsection{One dimensional Euler equations}

The one dimensional Euler's equations are given by

$$
U_{t}+F(U)_{x}=0
$$

where $U=(\rho, \rho u, e)^{T}$, and $F(U)=\left(\rho u, \rho u^{2}+p, u(e+p)\right)^{T}$. Here $\rho$ is the density, $u$ is the velocity, $e$ is the total energy, $p$ is the pressure, and for ideal gas $e=\frac{p}{\gamma-1}+\frac{1}{2} \rho u^{2}, \gamma=1.4$ is the ratio of specific heat.

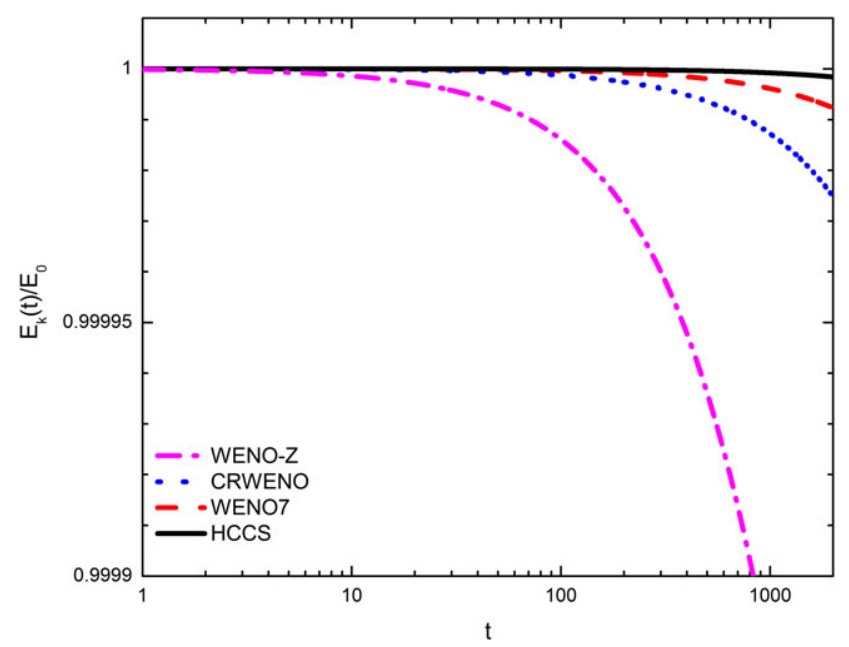

Figure 6. Integrated kinetic energy evolution of the vortex convection problem. [Colour figure can be viewed at wileyonlinelibrary.com]

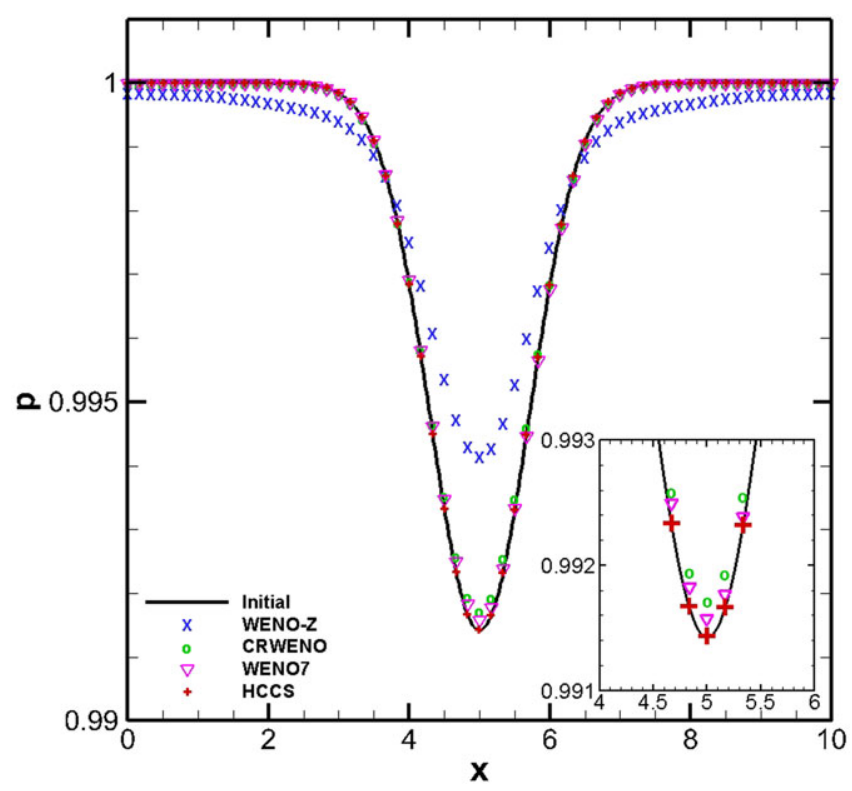

Figure 7. Pressure distributions at $y=5$ for different schemes at $t=2000$. [Colour figure can be viewed at wileyonlinelibrary.com] 
Four typical examples that contain strong discontinuities are considered here. The first example is the Sod problem. The initial condition is as follows:

$$
(\rho, u, p)= \begin{cases}(1,0,1) & x \leqslant 0 \\ (0.125,0,0.1) & x>0\end{cases}
$$

with zero gradient boundary conditions applied at $x= \pm 0.5$.

The second problem is the Lax problem, the initial condition is given by

$$
(\rho, u, p)= \begin{cases}(0.445,0.698,3.528) & x \leqslant 0 \\ (0.5,0,0.571) & x>0\end{cases}
$$

with zero gradient boundary conditions at $x= \pm 5$.

The third problem is the Shu-Osher problem. It describes the interaction of a Mach 3 shock with a density wave. The initial condition is given by

$$
(\rho, u, p)= \begin{cases}\left(\frac{27}{7}, \frac{4 \sqrt{35}}{9}, \frac{31}{3}\right) & x<-4 \\ \left(1+\frac{1}{5} \sin 5 x, 0,1\right) & x \geqslant-4\end{cases}
$$

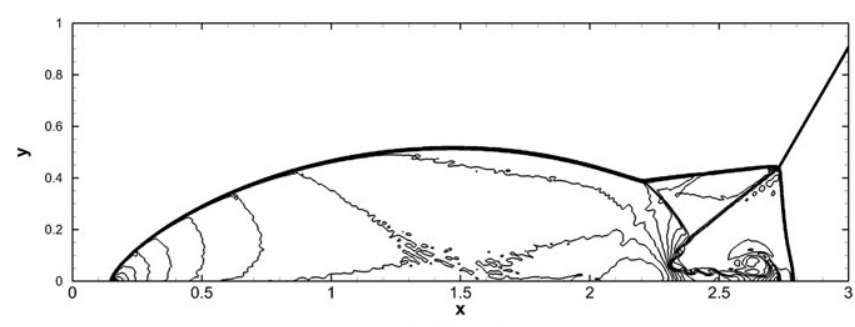

(a) WENO-Z
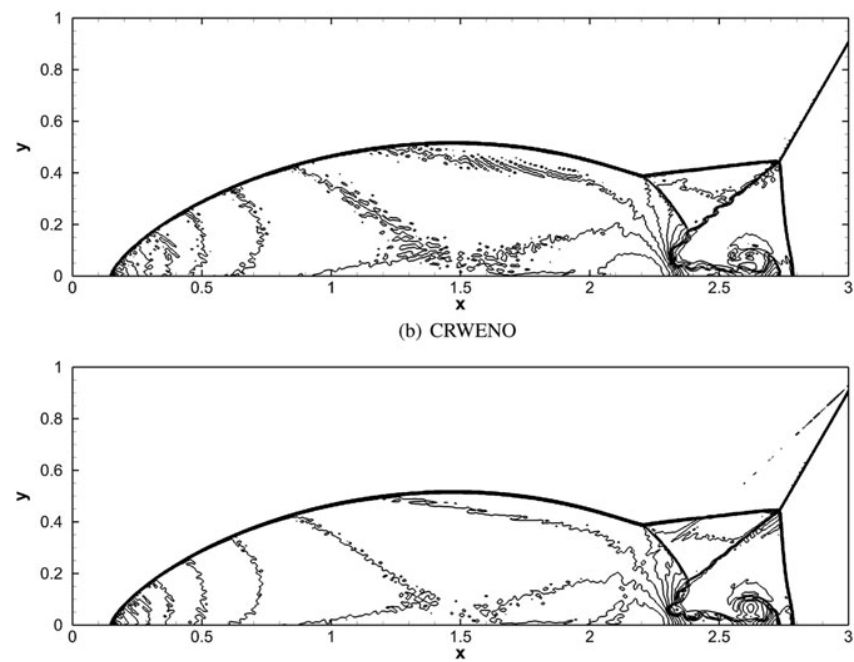

(c) WENO7

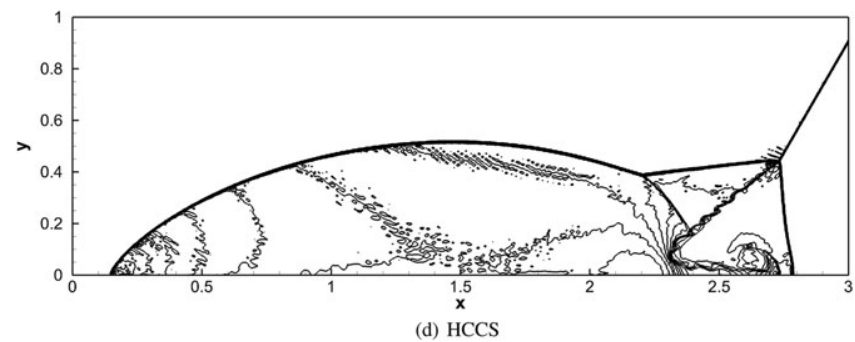

Figure 8. Density contours of the double Mach reflection problem at $t=0.2$, ranging from $\rho=2.1793$ to 24 with 30 equally separated levels, with gird number $960 \times 240$. 
Zero gradient boundary conditions are applied at $x= \pm 5$.

The zero gradient boundary condition $\left.\frac{\partial f}{\partial n}\right|_{i}=0$ is given by

$$
f_{i}=\left(4 f_{i-1}-f_{i-2}\right) / 3
$$

at right boundary points $i=(N-2, N-1, N)$ and

$$
f_{i}=4 f_{i+1}-3 f_{i+2}
$$

at left boundary points $i=(0,1,2)$.

The last problem is the interacting blast waves case. The initial condition is given by

$$
(\rho, u, p)=\left\{\begin{array}{cc}
(1,0,1000) & 0 \leqslant x<0.1 \\
(1,0,0.01) & 0 \leqslant x<0.9 \\
(1,0,100) & 0.9 \leqslant x \leqslant 1 .
\end{array}\right.
$$

Reflecting boundary conditions are set at boundaries.

All cases are computed with $N=200$. Reference results are obtained by the CRWENO scheme with 2000 points. Figure 5 illustrates results of the aforementioned cases. It can be seen that HCCS maintains the non-oscillatory property of the CRWENO scheme for strong shocks and that the

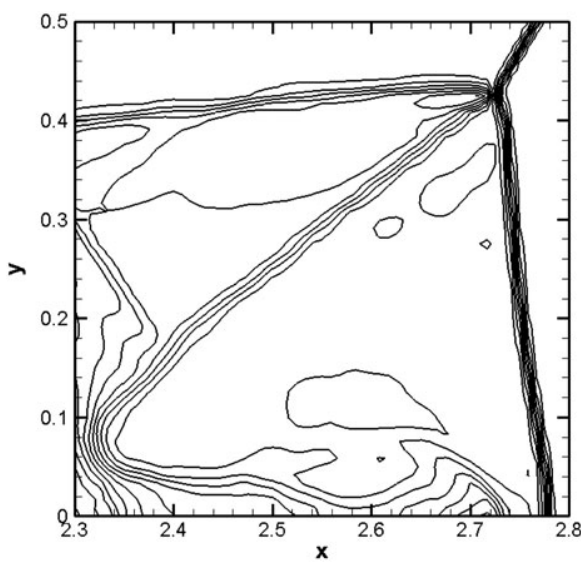

(a) WENO-Z

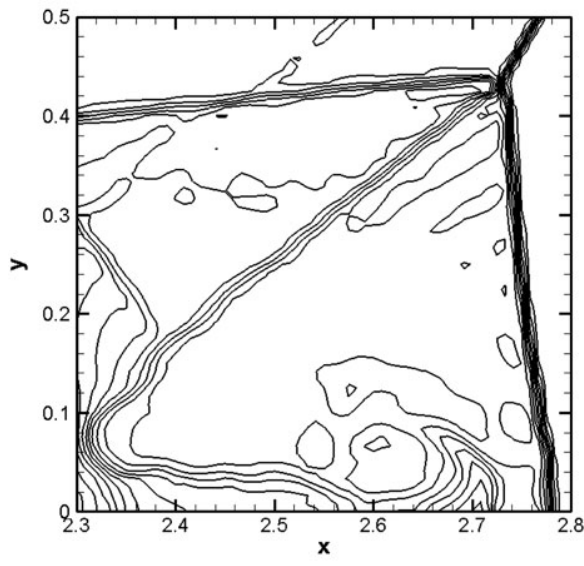

(c) WENO7

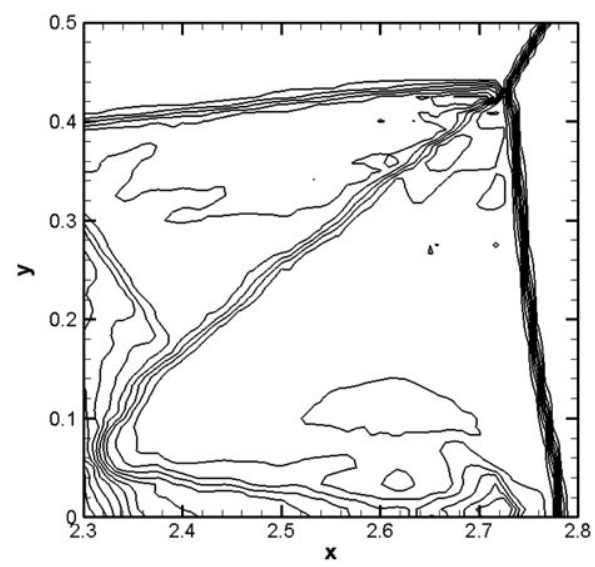

(b) CRWENO

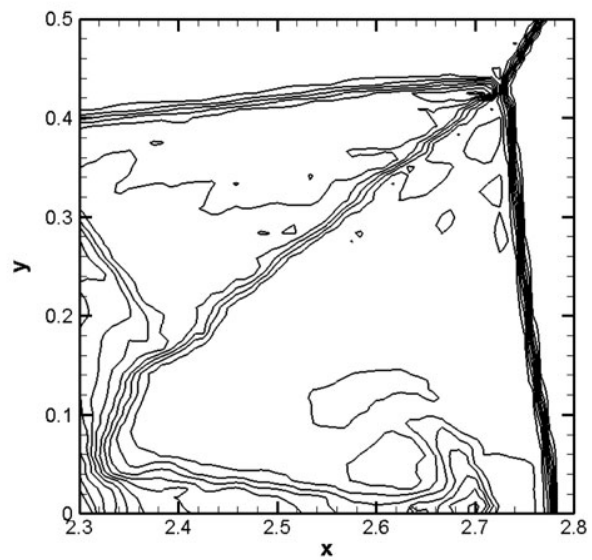

(d) HCCS

Figure 9. Density contours of the uprolling region at $t=0.2$, ranging from $\rho=1.3999$ to 24 with 30 equally separated levels, with gird number $480 \times 120$. 
hybriding method (24) does not introduce non-physical oscillations. Besides, HCCS shows better resolution than other methods for smooth short waves for the Shu-Osher problem.

\subsection{Two dimensional Euler equations}

The two dimensional Euler equations of ideal gas are given by

$$
U_{t}+F_{x}+G_{y}=0,
$$

where $U, F, G$ vectors are written as follows:

$$
U=\left[\begin{array}{l}
\rho \\
\rho u \\
\rho v \\
e
\end{array}\right], F=\left[\begin{array}{l}
\rho u \\
\rho u^{2}+p \\
\rho u v \\
e u+p u
\end{array}\right], G=\left[\begin{array}{l}
\rho v \\
\rho u v \\
\rho v^{2}+p \\
e v+p v
\end{array}\right] .
$$

In this section, two problems, the isentropic vortex convection problem and the double Mach reflection problem, are simulated with the WENO-Z scheme, the CRWENO scheme, the seventh-order WENO scheme, and HCCS.

5.3.1. Isentropic vortex convection. This problem was suggested to test the dissipation property of a certain scheme [39]. An isentropic vortex moves across the computational domain periodically.

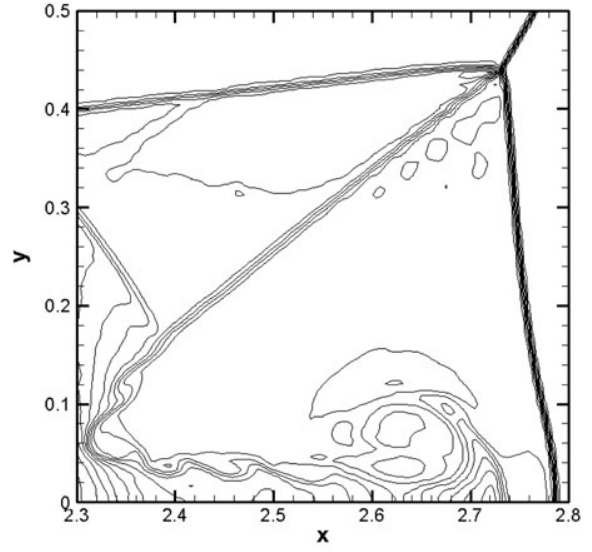

(a) WENO-Z

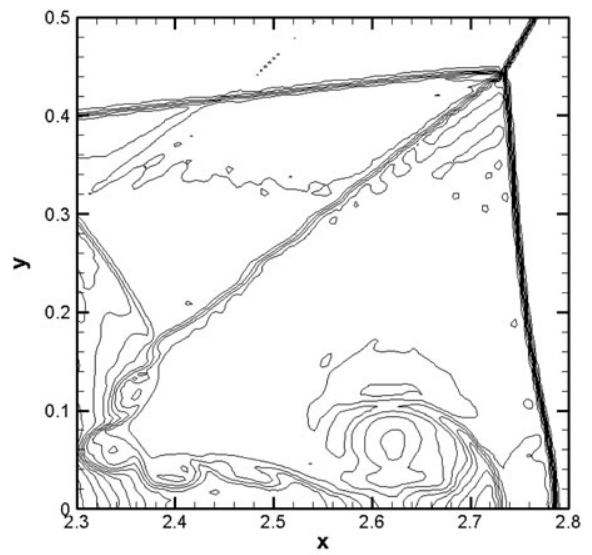

(c) WENO7

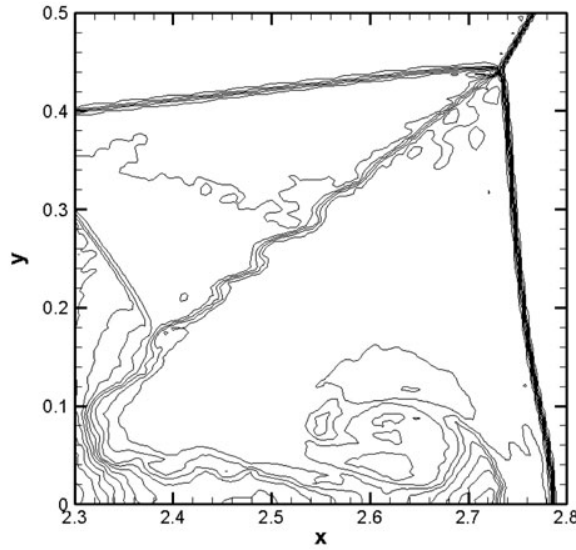

(b) CRWENO

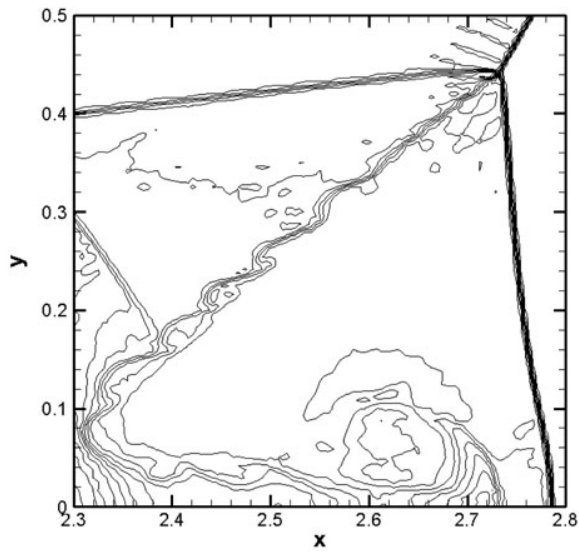

(d) HCCS

Figure 10. Density contours of the uprolling region at $t=0.2$, ranging from $\rho=1.3999$ to 24 with 30 equally separated levels, with gridd number $960 \times 240$. 
The computational domain is $[0,10] \times[0,10]$ with the free stream conditions specified as $\rho_{\infty}=$ $1, u_{\infty}=0.5, v_{\infty}=0, p_{\infty}=1$. The initial flow conditions are given by

$$
\begin{gathered}
\rho=\left[1-\frac{(\gamma-1) b^{2}}{8 \gamma \pi^{2}} e^{1-r^{2}}\right]^{\frac{1}{\gamma-1}} ; p=\rho^{\gamma} \\
\delta u=-\frac{b}{2 \pi} e^{\frac{1-r^{2}}{2}}\left(y-y_{c}\right) \\
\delta v=\frac{b}{2 \pi} e^{\frac{1-r^{2}}{2}}\left(x-x_{c}\right),
\end{gathered}
$$

where $r^{2}=\left(x-x_{c}\right)^{2}+\left(y-y_{c}\right)^{2}$ is the distance from the vortex center $\left(x_{c}, y_{c}\right)=(5,5)$, and the vortex strength $b$ is 0.5 . Periodic boundary conditions are applied to all boundaries. Dissipation is measured by instantaneous integrated kinetic energy over the computational domain: $E_{k}=\sum_{i j} 0.5 \rho_{i j}\left(u_{i j}^{2}+v_{i j}^{2}\right)$. The grid size is set to $N_{x} \times N_{y}=61 \times 61$, and solution is integrated to $t=2000$.

Figure 6 shows the evolution of the integrated kinetic energy over the computational domain. As the Euler equations are essentially inviscid, the damping of kinetic energy indicates the dissipation caused by numerical methods. Less damping of HCCS implies that HCCS has very low dissipation.

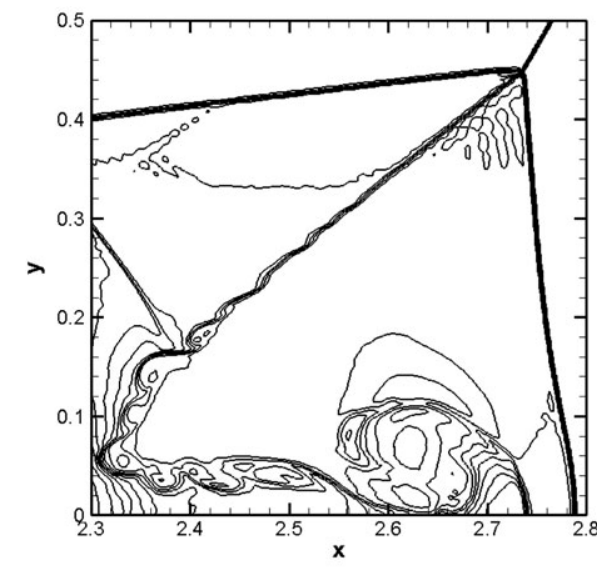

(a) WENO-Z

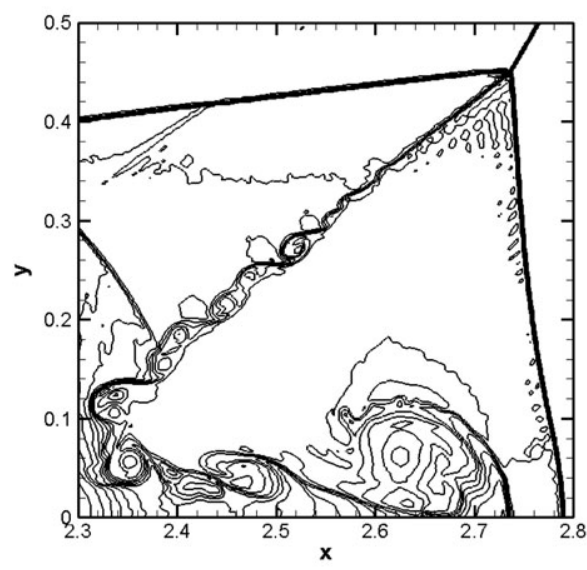

(c) WENO7

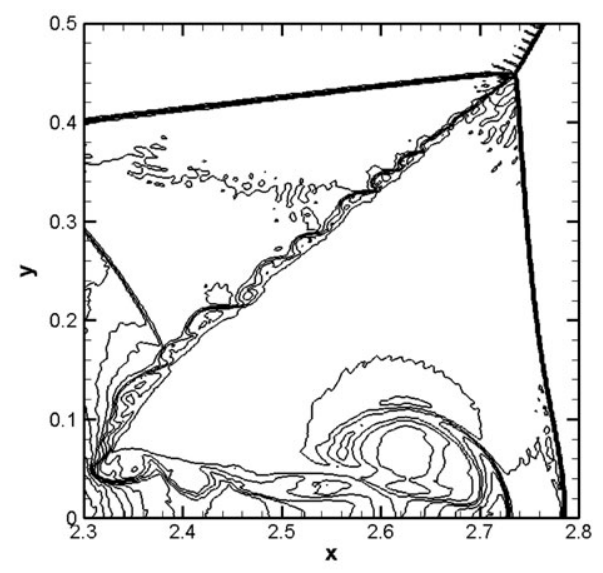

(b) CRWENO

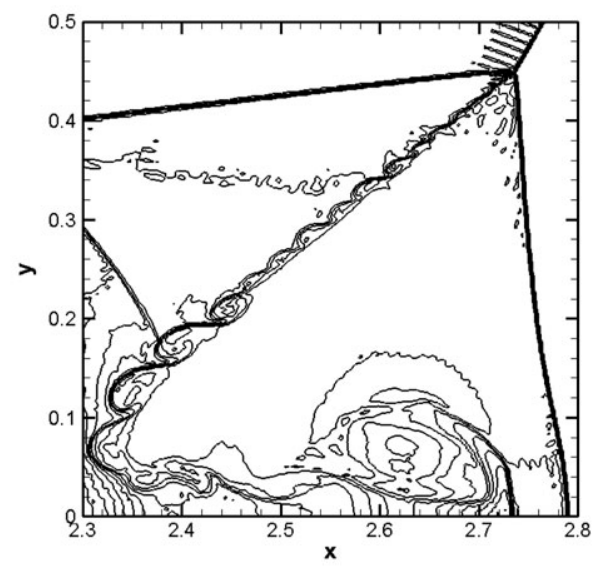

(d) HCCS

Figure 11. Density contours of the uprolling region at $t=0.2$, ranging from $\rho=1.3999$ to 24 with 30 equally separated levels, with gridd number $1920 \times 480$. 
The pressure distributions at $t=2000$ given in Figure 7 shows that HCCS obtains almost identical pressure as the initial profile while other schemes show apparent error. Besides, the results given by other schemes become asymmetric; however, the exact solution is symmetric. HCCS can keep the symmetric structure of the solution. This low dissipation and symmetric maintaining property of HCCS is very improtant for Direct Numerical Simulation (DNS) of turbulent flow. Even if very small numerical dissipation of the scheme being used in DNS may affect the physical dissipation and convection process [18].

5.3.2. Double Mach reflection. The double Mach reflection test is a mimic of planar shock reflection from a wedge. It has been extensively simulated to test the ability of shock-capturing and small-scale structure resolution of a certain scheme. In the present simulation, the computational domain is $[0,4] \times[0,1]$. The lower boundary is set to be reflecting wall starting from $x=\frac{1}{6}$. At $t=0$, a right-moving $60^{\circ}$ inclined Mach 10 shock is positioned at $\left(\frac{1}{6}, 0\right)$. The upper boundary is set to describe the exact motion of the Mach 10 shock:

$$
(\rho, u, v, p)= \begin{cases}\left(8,8.25 \frac{\sqrt{3}}{2},-8.25 \frac{1}{2}, 116.5\right), & x \leqslant \frac{1}{6}+\frac{1}{\sqrt{3}}(1+20 t) \\ (1.4,0,0,1), & x>\frac{1}{6}+\frac{1}{\sqrt{3}}(1+20 t) .\end{cases}
$$

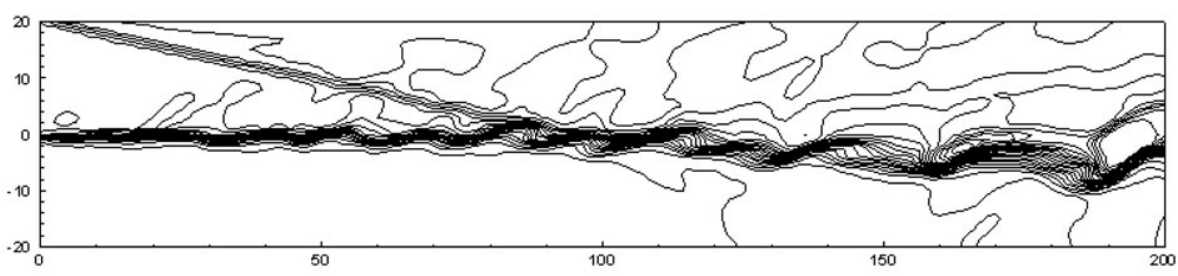

(a) WENO-Z

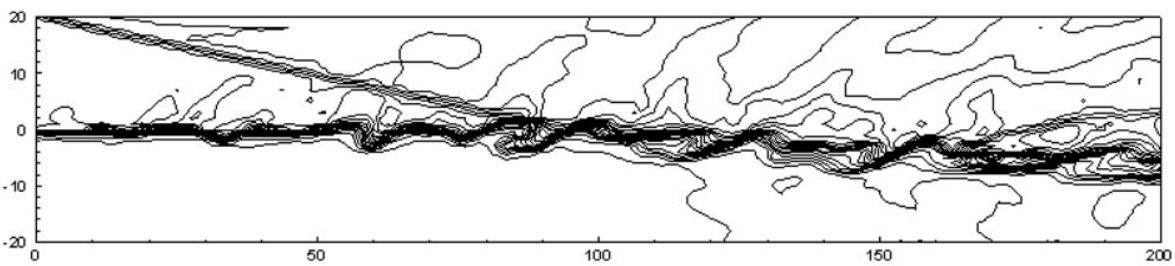

(b) CRWENO

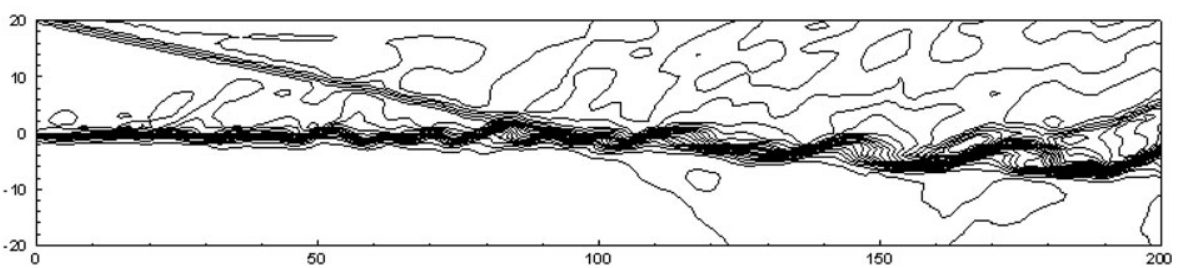

(c) WENO7

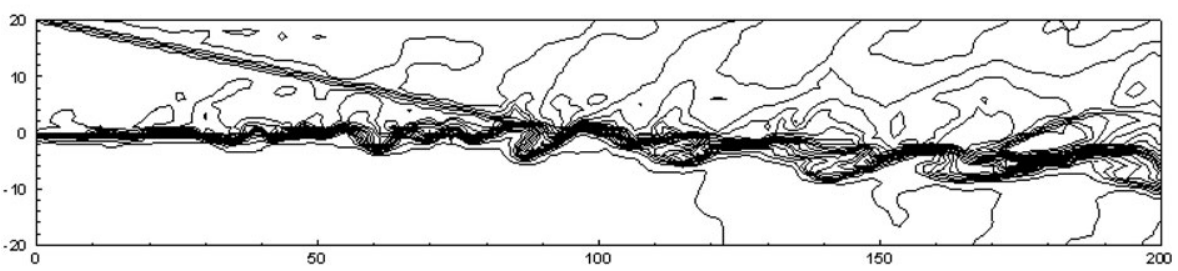

(d) HCCS

Figure 12. Density isolines of Shock/Shear layer interaction solutions at $t=120$, coarse grid $N_{x} \times N_{y}=$ $161 \times 41$. 
The left boundary at $x=0$ is assigned with post-shock values. Outflow conditions with zero gradients $\frac{\partial f}{\partial x}=0$ are set at $x=4$. Detailed description of the double Mach reflection test can be found in [40]. Three grids are used in this simulation: $N_{x} \times N_{y}=480 \times 120, N_{x} \times N_{y}=960 \times 240$, and $N_{x} \times N_{y}=1920 \times 480$.

Density isolines obtained the $960 \times 240$ grid by different schemes are show in Figure 8 . Shock structures are well captured by all schemes. The zoomed uprolling regions are shown in Figure 9-11. On finer grids, the contact instability structures can be better resolved. It can be seen that the compact schemes, including CRWENO and HCCS schemes, obtain richer small structures caused by Kelvin-Helmholtz instabilities than WENO-Z and WENO7. HCCS shows the best small-scale structure resolution along the contact discontinuity line on each grid.

\subsection{Two dimensional Navier-Stokes equations}

The two dimensional Navier-Stokes equations for ideal gas are given by

$$
U_{t}+F_{x}+G_{y}=\frac{1}{R e} F_{x}^{v}+\frac{1}{R e} G_{y}^{v}
$$

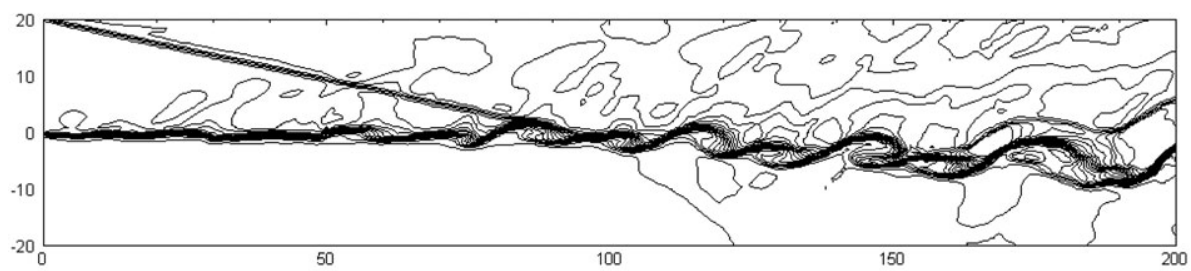

(a) WENO-Z

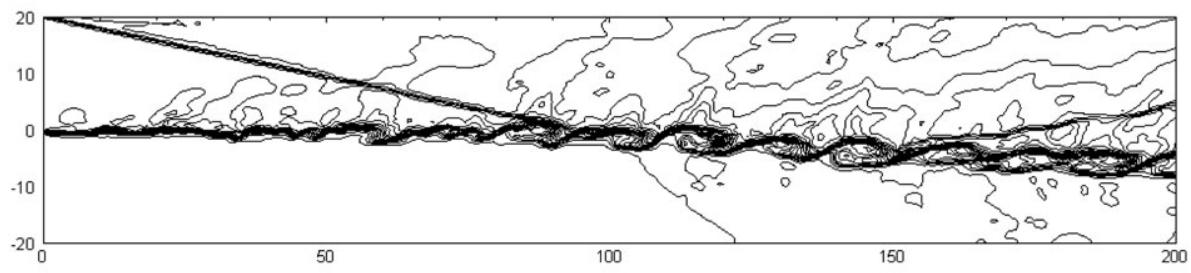

(b) CRWENO

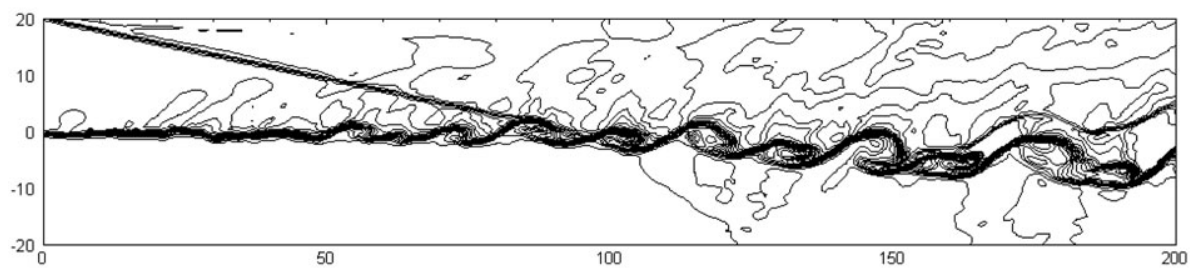

(c) WENO7

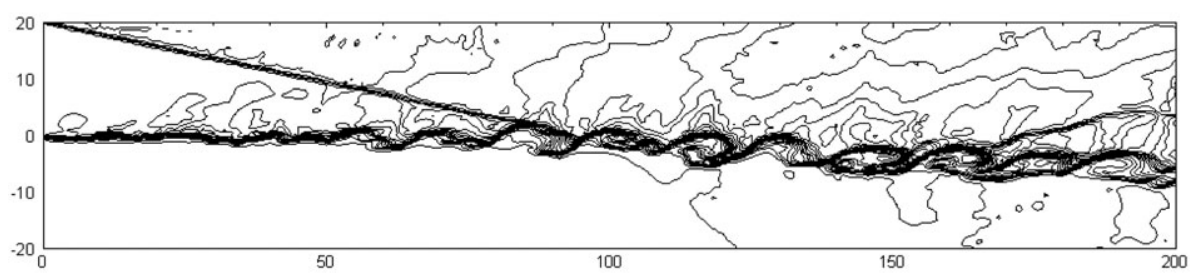

(d) HCCS

Figure 13. Density isolines of Shock/Shear layer interaction solutions at $t=120$, medium grid $N_{x} \times N_{y}=$ $321 \times 81$. 
where $U, F, G$, vectors are the same as given in (39), and $F_{v}, G_{v}$ are given by

$$
F^{v}=\left[\begin{array}{c}
0 \\
\tau_{x x} \\
\tau_{x y} \\
u \tau_{x x}+v \tau_{x y}+q_{x}
\end{array}\right], G^{v}=\left[\begin{array}{c}
0 \\
\tau_{y x} \\
\tau_{y y} \\
u \tau_{y x}+v \tau_{y y}+q_{y}
\end{array}\right]
$$

in which

$$
\begin{aligned}
\tau_{x x} & =\mu\left(\frac{4}{3} \frac{\partial u}{\partial x}-\frac{2}{3} \frac{\partial v}{\partial y}\right), \tau_{y y}=\mu\left(\frac{4}{3} \frac{\partial v}{\partial y}-\frac{2}{3} \frac{\partial u}{\partial x}\right), \\
\tau_{x y} & =\tau_{y x}=\mu\left(\frac{\partial u}{\partial y}+\frac{\partial v}{\partial x}\right), \\
q_{x} & =\mu \frac{1}{(\gamma-1) M^{2} \operatorname{Pr}} \frac{\partial T}{\partial x}, \\
q_{y} & =\mu \frac{1}{(\gamma-1) M^{2} \operatorname{Pr}} \frac{\partial T}{\partial y} .
\end{aligned}
$$

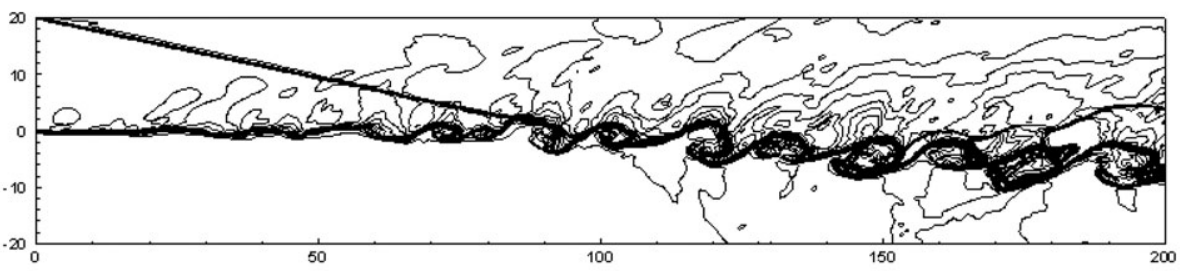

(a) WENO-Z

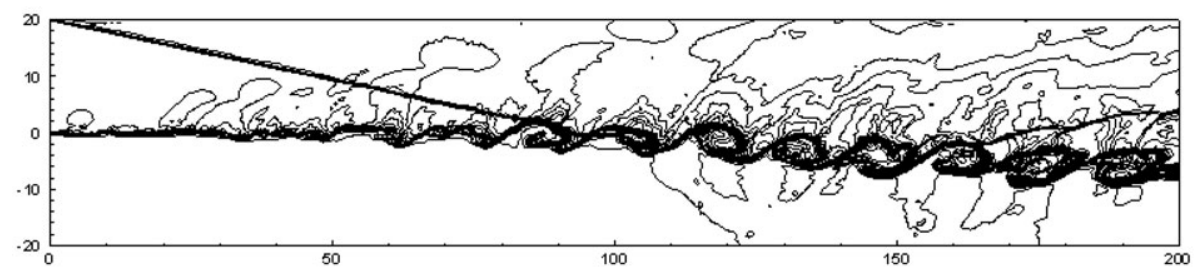

(b) CRWENO

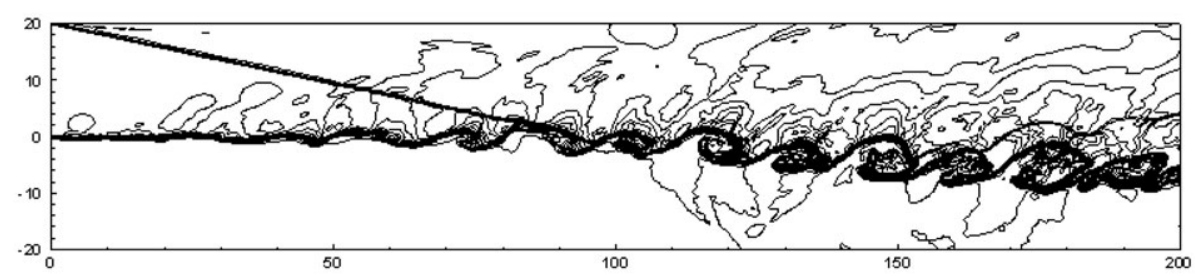

(c) WENO7

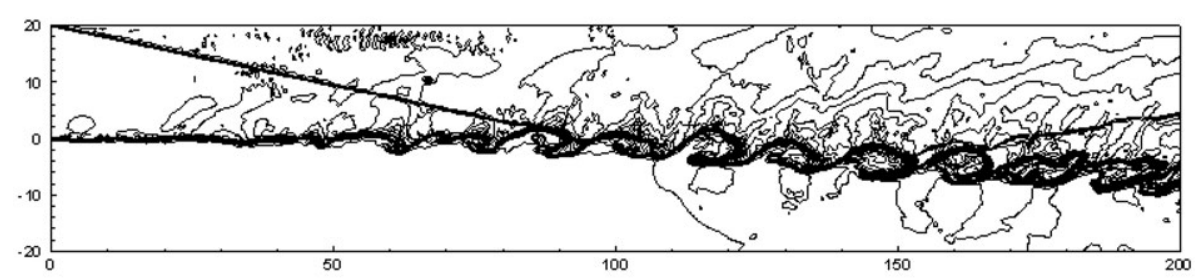

(d) HCCS

Figure 14. Density isolines of Shock/Shear layer interaction solutions at $t=120$, fine grid $N_{x} \times N_{y}=$ $641 \times 161$. 
The viscous terms are discretized with the fourth-order central difference scheme [41].

The shock/shear layer interaction problem is designed to measure the resolution of schemes [42]. An oblique shock impacts on a Mach 0.6 shear layer and is reflected by a wall at the lower boundary. The computational domain is $[0,200] \times[-20,20]$. A coarse grid $N_{x} \times N_{y}=161 \times 41$, a medium grid $N_{x} \times N_{y}=321 \times 81$, and a fine grid $N_{x} \times N_{y}=641 \times 161$ are used in the simulation. At $x=0$, the inlet condition is specified as

$$
u=2.5+0.5 \tanh (2 y)
$$

for the upper stream $(y>0), \rho=1.6374, p=0.3327$ and for the lower stream $(y<0), \rho=$ $0.3626, p=0.3327$. Post shock conditions are set at the upper boundary, and a slip wall condition is applied at the lower boundary. Fluctuations are added to the vertical velocity component:

$$
\begin{aligned}
& v^{\prime}=\sum_{k=1}^{2} a_{k} \cos \left(2 \pi k t / T+\phi_{k}\right) \exp \left(-y^{2} / b\right), \\
& b=10, a_{1}=a_{2}=0.05, \phi_{1}=0, \phi_{2}=\pi / 2,
\end{aligned}
$$

in which $T=\lambda / u_{c}$ is the period, $\lambda=30$ is the wavelength, and $u_{c}=2.68$ is the convective velocity. The Prandtl number $P r$ is set to 0.72 , and the Reynolds number $R e$ is chosen to be 500 .

Solutions at $t=120$ are shown in Figure 12-14 for each grid. All schemes can capture shock waves well. The vortex structures calculated by the compact schemes (i.e., CRWENO and HCCS) and the explicit schemes (i.e., WENO-Z and WENO7) are different. The explicit schemes smear the shear layer, while the compact schemes can well capture the vortices induced by shear layer instability. Note that the shear layer fluctuations grow and affect the shear layer structure. Better resolution of the shear layer growth indicates that the instability growing process is captured more accurately. Compared with CRWENO, HCCS resolves slightly less smeared shear layer before it interacts with shock on both coarse and fine grids. After the shear layer interacts with the shock, the shear layer vortex structures calculated by different schemes are different because of different upstream structure. Near the outflow boundary, the reflecting shock over the shear layer calculated by HCCS is less smeared than CRWENO on the coarse grid and the medium grid. On the fine grid, the reflecting shock structure given by HCCS and CRWENO is almost the same, which implies that this grid is fine enough to resolve these structures.

\section{CONCLUSION}

In this paper, a problem-independent weighting switch function for developing high order hybrid schemes is proposed. The function approaches 1 with high order accuracy in smooth regions and zero in vicinity of discontinuities. By using the switch function, a seventh-order hybrid compactCRWENO scheme on the 5-points WENO stencil is constructed. Analysis and numerical tests show that the new hybrid scheme is seventh-order accurate in smooth regions and has good shockcapturing capability, and its low dissipation and low computational costs imply that HCCS is more efficient than the seventh-order WENO scheme.

Moreover, the idea of the switch function can be easily extended to develop higher order function for high-order hybrid schemes. Compared with those switch functions used in literatures, the present one is problem independent. Hence, hybrid schemes developed with the new method are more robust and can be applied to a wide range of complex flow simulations.

\section{ACKNOWLEDGEMENTS}

This work was supported by NSFC (No. 11272325 and No. 11272324) and NSAF (No. U1530145). The authors acknowledge the anonymous reviewers for their valuable comments. 


\section{REFERENCES}

1. Liu XD, Osher S, Chan T. Weighted essentially non-oscillatory schemes. Journal of Computational Physics 1994; 115(1):200-212.

2. Jiang GS, Shu CW. Efficient implementation of weighted Eno schemes. Journal of Computational Physics 1996; 126(1):202-228.

3. Henrick AK, Aslam TA, Powers JM. Mapped weighted essentially non-oscillatory schemes: achieving optimal order near critical points. Journal of Computational Physics 2005; 207(2):542-567.

4. Borges R, Carmona M, Costa B, Don WS. An improved weighted essentially non-oscillatory scheme for hyperbolic conservation laws. Journal of Computational Physics 2008; 227(6):3191-3211.

5. Castro M, Costa B, Don WS. High order weighted essentially non-oscillatory WENO-Z schemes for hyperbolic conservation laws. Journal of Computational Physics March 2011; 230(5):1766-1792.

6. Yamaleev NK, Carpenter MH. A systematic methodology for constructing high-order energy stable WENO schemes. Journal of Computational Physics June 2009; 228(11):4248-4272.

7. Yamaleev NK, Carpenter MH. Third-order energy stable WENO scheme. Journal of Computational Physics May 2009; 228(8):3025-3047.

8. Fan P, Shen Y, Tian B, Yang C. A new smoothness indicator for improving the weighted essentially non-oscillatory scheme. Journal of Computational Physics July 2014; 269(C):329-354.

9. Shen Y, Zha G. Improvement of weighted essentially non-oscillatory schemes near discontinuities. Computers \& Fluids June 2014; 96(C):1-9.

10. Shen Y, Liu L, Yang Y. Multistep weighted essentially non-oscillatory scheme. International Journal for Numerical Methods in Fluids February 2014; 75(4):231-249.

11. Peng J, Shen Y. Improvement of weighted compact scheme with multi-step strategy for supersonic compressible flow. Computers \& Fluids July 2015; 115(0):243-255.

12. Balsara DS, Shu CW. Monotonicity preserving weighted essentially non-oscillatory schemes with increasingly high order of accuracy. Journal of Computational Physics 2000; 160(2):405-452.

13. Gerolymos GA, Sénéchal D, Vallet I. Very-high-order WENO schemes. Journal of Computational Physics December 2009; 228(23):8481-8524.

14. Martin MP, Taylor EM, Wu M, Weirs VG. A bandwidth-optimized WENO scheme for the effective direct numerical simulation of compressible turbulence. Journal of Computational Physics 2006; 220(1):270-289.

15. Pirozzoli S. On the spectral properties of shock-capturing schemes. Journal of Computational Physics 2006; 219(2):489-497.

16. Zhao S, Lardjane N, Fedioun I. Comparison of improved finite-difference WENO schemes for the implicit large eddy simulation of turbulent non-reacting and reacting high-speed shear flows. Computers \& Fluids 2014; 95:74-87.

17. Jia F, Gao Z, Don W. A spectral study on the dissipation and dispersion of the WENO schemes. Journal of Scientific Computing 2014; 63(1):49-77.

18. Johnsen E, Larsson J, Bhagatwala AV, Cabot WH, Moin P, Olson BJ, Rawat PS, Shankar SK, Sjögreen B, Yee H, et al. Assessment of high-resolution methods for numerical simulations of compressible turbulence with shock waves. Journal of Computational Physics 2010; 229(4):1213-1237.

19. Lele SK. Compact finite difference schemes with spectral-like resolution. Journal of Computational Physics 1992; 103(1):16-42.

20. Deng X, Maekawa H. Compact high-order accurate nonlinear schemes. Journal of Computational Physics 1997; 130(1):77-91.

21. Jiang L, Shan H, Liu C. Weighted compact scheme for shock capturing. International Journal of Computational Fluid Dynamics 2001; 15(2):147-155.

22. Ghosh D, Baeder JD. Compact reconstruction schemes with weighted ENO limiting for hyperbolic conservation laws. SIAM Journal on Scientific Computing 2012; 34(3):A1678-A1706.

23. Adams NA, Shariff K. A high-resolution hybrid compact-Eno scheme for shock-turbulence interaction problems. Journal of Computational Physics. 1996; 127(1):27-51.

24. Pirozzoli S. Conservative hybrid compact-WENO schemes for shock-turbulence interaction. Journal of Computational Physics 2002; 178(1):81-117.

25. Ren YX, Liu M, Zhang H. A characteristic-wise hybrid compact-WENO scheme for solving hyperbolic conservation laws. Journal of Computational Physics 2003; 192(2):365-386.

26. Hill DJ, Pullin DI. Hybrid tuned center-difference-WENO method for large eddy simulations in the presence of strong shocks. Journal of Computational Physics 2004; 194(2):435-450.

27. Costa B, Don WS. Multi-domain hybrid spectral-WENO methods for hyperbolic conservation laws. Journal of Computational Physics June 2007; 224(2):970-991.

28. Cheng J, Lu Y, Liu T. Multidomain hybrid RKDG and WENO methods for hyperbolic conservation laws. SIAM: SIAM Journal on Scientific Computing April 2013; 35(2):A1049-A1072.

29. Ziegler JL, Deiterding R, Shepherd JE, Pullin DI. An adaptive high-order hybrid scheme for compressive, viscous flows with detailed chemistry. Journal of Computational Physics August 2011; 230(20):7598-7630.

30. Gao Z, Don WS. Mapped hybrid central-WENO finite difference scheme for detonation waves simulations. Journal of Computational Physics August 2012; 55(2):351-371.

31. Shen Y, Zha G. Generalized finite compact difference scheme for shock/complex flowfield interaction. Journal of Computational Physics June 2011; 230(12):4419-4436. 
32. Li G, Qiu J. Hybrid weighted essentially non-oscillatory schemes with different indicators. Journal of Computational Physics October 2010; 229(21):8105-8129.

33. Kim D, Kwon JH. A high-order accurate hybrid scheme using a central flux scheme and a WENO scheme for compressible flowfield analysis. Journal of Computational Physics 2005; 210(2):554-583.

34. Zhou Q, He F, Shen MY. A family of efficient high-order hybrid finite difference schemes based on WENO schemes. International Journal of Computational Fluid Dynamics April 2012; 26(4):205-229.

35. Ghosh D, Baeder JD. Weighted non-linear compact schemes for the direct numerical simulation of compressible turbulent flows. Journal of Scientific Computing 2014; 61(1):61-89.

36. Shen Y, Zha G. A Robust seventh-order WENO scheme and ITS applications. 46th AIAA Aerospace Sciences Meeting and Exhibit, American Institute of Aeronautics and Astronautics, Reston, Virigina, 2012; 1-22.

37. Shu CW. Total-variation-diminishing time discretizations. SIAM Journal on Scientific and Statistical Computing 1988; 9(6): 1073-1084.

38. Steger SL, Warming RF. Flux vector splitting of the inviscid gasdynamic equations with application to finitedifference methods. Journal of Computational Physics 1981; 40(2):263-293.

39. Davoudzadeh F, McDonald H, Thompson BE. Accuracy evaluation of unsteady cfd numerical schemes by vortex preservation. Computers \& Fluids 1995; 24(8):883-895.

40. Woodward P, Colella P. The numerical simulation of two-dimensional fluid flow with strong shocks. Journal of Computational Physics 1984; 54(1):115-173.

41. Shen Y, Zha G, Chen X. High order conservative differencing for viscous terms and the application to vortex-induced vibration flows. Journal of Computational Physics 2009; 228(22):8283-8300.

42. Yee HC, Sandham ND, Djomehri MJ. Low-dissipative high-order shock-capturing methods using characteristicbased filters. Journal of Computational Physics 1999; 150(1):199-238. 